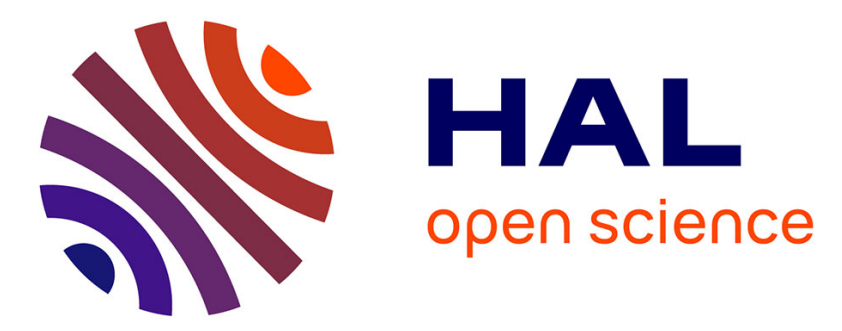

\title{
Convergence and performance of the peeling wavelet denoising algorithm
}

Céline Lacaux, Aurélie Muller, Radu Ranta, Samy Tindel

\section{To cite this version:}

Céline Lacaux, Aurélie Muller, Radu Ranta, Samy Tindel. Convergence and performance of the peeling wavelet denoising algorithm. Metrika, 2014, 77 (4), pp.509-537. 10.1007/s00184-013-0451-y . hal-00903593

\section{HAL Id: hal-00903593 \\ https://hal.science/hal-00903593}

Submitted on 12 Nov 2013

HAL is a multi-disciplinary open access archive for the deposit and dissemination of scientific research documents, whether they are published or not. The documents may come from teaching and research institutions in France or abroad, or from public or private research centers.
L'archive ouverte pluridisciplinaire HAL, est destinée au dépôt et à la diffusion de documents scientifiques de niveau recherche, publiés ou non, émanant des établissements d'enseignement et de recherche français ou étrangers, des laboratoires publics ou privés. 


\title{
CONVERGENCE AND PERFORMANCE OF THE PEELING WAVELET DENOISING ALGORITHM
}

\author{
CÉLINE LACAUX, AURÉLIE MULLER-GUEUDIN, RADU RANTA, AND SAMY TINDEL
}

\begin{abstract}
This note is devoted to an analysis of the so-called peeling algorithm in wavelet denoising. Assuming that the wavelet coefficients of the useful signal are modeled by generalized Gaussian random variables and its noisy part by independent Gaussian variables, we compute a critical thresholding constant for the algorithm, which depends on the shape parameter of the generalized Gaussian distribution. We also quantify the optimal number of steps which have to be performed, and analyze the convergence of the algorithm. Several implementations are tested against classical wavelet denoising procedures on benchmark and simulated biological signals.
\end{abstract}

\section{INTRODUCTION}

Among the wide range of applications of wavelet theory which have emerged during the last 20 years, the processing of noisy signals is certainly one of the most important one. In general, denoising is performed by thresholding and/or shrinkage algorithms, as discussed and analysed for example in [7], in the application oriented presentation [1] or by the sharp uniform central limit theorems in [8]. This fundamental algorithm can be summarized in the following way: recall that the wavelet decomposition of a function $z \in L^{2}(\mathbb{R})$ is usually written as:

$$
z(t)=\sum_{k=0}^{2^{j_{0}}-1} \alpha_{j_{0} k} \phi_{j_{0} k}(t)+\sum_{j=j_{0}}^{\infty} \sum_{k=0}^{2^{j}-1} \beta_{j k} \psi_{j k}(t),
$$

where the coefficients $\alpha, \beta$ are obtained by projection in $L^{2}(\mathbb{R})$ :

$$
\alpha_{j_{0} k}=\left\langle\phi_{j_{0} k}, z\right\rangle_{L^{2}(\mathbb{R})}, \quad \text { and } \quad \beta_{j k}=\left\langle\psi_{j k}, z\right\rangle_{L^{2}(\mathbb{R})} .
$$

The functions $\psi$ and $\phi$ are respectively called mother and father wavelets (or, alternatively, wavelets and scaling functions respectively), and enjoy some suitable scaling and algebraic properties (see e.g. [5, 12] for a complete account on wavelet decompositions). In this context, the thresholding algorithm assumes that, if $z$ can be decomposed into $z=x+w$, where $x$ is the useful signal and $w$ its noisy part, then the wavelet coefficients corresponding to $w$ will typically be very small. A reasonable estimation for the signal $x$ is thus:

$$
\hat{x}(t)=\sum_{k=0}^{2^{j_{0}}-1} \alpha_{j_{0} k} \mathbf{1}_{\left\{\left|\alpha_{j_{0}}\right| \geq \tau\right\}} \phi_{j_{0} k}(t)+\sum_{j=j_{0}}^{J} \sum_{k=0}^{2^{j}-1} \beta_{j k} \mathbf{1}_{\left\{\left|\beta_{j k}\right| \geq \tau\right\}} \psi_{j k}(t),
$$

Date: November 12, 2013.

2000 Mathematics Subject Classification. 62G08,62G20.

Key words and phrases. Wavelets, denoising, peeling algorithm, empirical processes, generalized Gaussian distribution.

C. Lacaux, A. Muller-Gueudin and S. Tindel are members of the BIGS (BIology, Genetics and Statistics) team at INRIA. 
where $\tau$ is a suitable threshold (which may also depend on the resolution $j$ and, in practice, except for very sparse signals, is often zero for the coefficients $\alpha_{j_{0}}$ of the scaling function) and where $J$ corresponds to the maximal resolution one is allowed to consider. It is then proved in the aforementioned references $[1,7]$ that this kind of estimator satisfies some nice properties concerning the asymptotic behavior of the approximation error, in terms of the total number of wavelet coefficients (which is denoted by $N$ in the sequel).

To properly fix the notations employed in this paper, note that continuous time functions $z, x$ and $w$ can be approximated, after sampling, by vectors containing their discrete time versions. Moreover, as the wavelet decomposition is linear, the model $z=x+w$ equally holds for the wavelet coefficients of the aforementioned functions. Therefore, in the sequel, we will denote by $z, x$ and $w$ respectively the wavelet coefficients of the measured signal, of the underlying noise-free signal and of the noise.

The literature on wavelet thresholding methods is very rich (see [1] for a review), but most of the algorithms consist in two steps: (i) compute $\tau$ starting from some hypothesis on the statistical properties of the wavelet coefficients and (ii) reconstruct the denoised signal according to (2).

The critical issue is thus the value of the threshold(s) $\tau$ : too low it is inefficient, too high it distorts the information from $x$. In order to improve the performances of wavelet-based denoising algorithms by adapting them to the processed signals, an iterative method called peeling algorithm has been introduced and shown to be particularly useful for biomedical applications in $[4,9]$.

The main algorithmic difference between peeling algorithms and classical methods consists in the threshold computing method: iterative for the former, one-step for the latter. Furthermore, peeling algorithms do not assume explicitly an underlying model $z=x+w$ (signal + noise, each one with its different statistical properties), but it focuses instead on the method used to estimate the threshold between large and small magnitude coefficients. Nevertheless, the two approaches are equivalent and the goal is strictly the same: separating large significant wavelet coefficients from small ones, attributed to noise.

With this equivalence in mind, the algorithm introduced in $[4,9]$ intends then to separate $x$ from $w$ iteratively. Indeed, the $k^{\text {th }}$ step of the procedure produces an estimated signal $\widehat{x}_{k}$, as well as an estimated noise $\widehat{w}_{k}$, initialized for $k=0$ as $\widehat{w}_{0}=z$. Then the $(k+1)^{\text {th }}$ step is as follows:

(1) Compute $\widehat{\sigma}_{k}^{2}=\frac{\left\|\widehat{w}_{k}\right\|^{2}}{N}$, where we recall that $N$ denotes the total number of wavelet coefficients involved in the analysis.

(2) Set a thresholding level $T_{k+1}$ as $T_{k+1}=h\left(\widehat{\sigma}_{k}\right)$, where $h$ is usually linear, which means that $T_{k+1}=F \widehat{\sigma}_{k}$ for a certain coefficient $F$.

(3) Compute $\Delta \widehat{x}_{k+1}$ as:

$$
\Delta \widehat{x}_{k+1}(q)=\widehat{w}_{k}(q) \mathbf{1}_{\left\{\left|\widehat{w}_{k}(q)\right| \geq T_{k+1}\right\}}
$$

for all the coefficients $q$ of the wavelet decomposition. The vectors $\widehat{x}_{k+1}, \widehat{w}_{k+1}$ are then defined as $\widehat{x}_{k+1}=\widehat{x}_{k}+\Delta \widehat{x}_{k+1}$, and $\widehat{w}_{k+1}=\widehat{w}_{k}-\Delta \widehat{x}_{k+1}$.

(4) Loop this procedure until a stop criterion of the form $\left\|\widehat{w}_{k}\right\|^{2}-\left\|\widehat{w}_{k+1}\right\|^{2} \leq \varepsilon$ is reached, for a certain positive constant $\varepsilon$. Notice that one can choose $\varepsilon=0$. 
This iterative procedure tends to retrieve a higher quantity of (approximate) signal $x$ from the noisy input $z$, correcting some of the failures of the original thresholding algorithm in some special situations.

On the basis of these promising experimental results, the peeling algorithm has been further investigated in $[14,15]$, and it has been first observed in those references that the peeling problem could be handled through a fixed point algorithm. This possibility stems basically from the fact that the sequence $\left\{T_{k} ; k \geq 0\right\}$ is decreasing (as $\left\|\widehat{w}_{k}\right\|^{2} \geq\left\|\widehat{w}_{k+1}\right\|^{2}$ ), which means that the previous algorithm can be reduced to the following:

(1) Set $T_{0}=+\infty$ and $T_{k+1}=f_{N}\left(T_{k}\right)$, where

$$
f_{N}(t)=F\left[\frac{\sum_{q \leq N} z^{2}(q) \mathbf{1}_{\{|z(q)|<t\}}}{N}\right]^{1 / 2},
$$

with $F$ a user chosen parameter that modifies the decreasing sequence $\left\{T_{k} ; k \geq 0\right\}$ and its final value at convergence $T_{f} \in[0,+\infty)$.

(2) Stop the loop when $T_{k+1}=T_{f}$, and then set

$$
\hat{x}(q)=z(q) \mathbf{1}_{\left\{|z(q)| \geq T_{f}\right\}} .
$$

The main issue is to ensure that the random threshold $T_{f}$ is positive almost surely. Under some hypothesis on the signal $z$, it is shown in [14] for $F$ large enough. Moreover, a further analysis on the choice of the coefficient $F$ is performed in [15].

However, in spite of the efforts made in the aforementioned references [14, 15], a probabilistic analysis of the algorithm is still missing. The current article proposes to make a step in this direction, and we proceed now to describe the results we have obtained.

First of all, our measured signal $z$ is of course characterized by the family of its wavelets coefficients, which will be denoted from now on by $\{z(q) ; q \leq N\}$. As said previously, most of the literature considers $z=x+w$ a sum between an ideal signal and the noise, generally presumed Gaussian. It is also quite usual to model the distribution of the wavelet coefficients of "real" signals or images as uncorrelated generalized Gaussian variables $[11,21,22,23,25]$. This hypothesis was empirically verified on several real images in $[24,23]$. In all these references, by "real" signal the authors designate the ideal $x$, while the wavelet coefficients of the noisy part are assumed independent Gaussian. In the current article, we take up this classical model of wavelet decomposition, and assume the following:

Hypothesis 1.1. Our signal $z=x+w$ can be decomposed as follows:

(1) The wavelet coefficients $\{x(q) ; q \leq N\}$ of the useful signal $x$ form an i.i.d family of generalized Gaussian variables, whose marginal density $\left(p_{\sigma, u}(t)\right)_{t \in \mathbb{R}}$ is given by

$$
p_{\sigma, u}(t)=\frac{\alpha}{\sigma} e^{-\frac{\mid \beta t u^{u}}{\sigma^{u}}}, \quad \text { with } \quad \beta=\left(\frac{\Gamma(3 / u)}{\Gamma(1 / u)}\right)^{1 / 2}, \quad \alpha=\frac{\beta u}{2 \Gamma(1 / u)},
$$

where $\Gamma$ stands for the usual Gamma function $\Gamma(\xi)=\int_{0}^{\infty} e^{-t} t^{\xi-1} d t$. Notice that the coefficient $\sigma>0$ above is the standard deviation of each random variable $x(q)$, and that $u>0$ represents the shape parameter of the probability law ( $u=2$ for the Gaussian, $u=1$ for the Laplace pdf). 
(2) The wavelet coefficients $\{w(q), q \leq N\}$ of the noisy part of the signal form an i.i.d. family of centered Gaussian variables, with standard deviation $\sigma_{w}$. Their common density is noted $p_{\sigma_{w}}$.

(3) The coefficients $\{x(q), q \leq N\}$ and $\{w(q), q \leq N\}$ are independent.

With these preliminary considerations in mind, the two main results which will be presented in this paper are the following:

(1) As shown in [14], the sequence of thresholds $\left\{T_{k} ; k \geq 0\right\}$ involved in the peeling algorithm converges almost surely. However, it is easily checked that it can converge either to a strictly positive quantity $T_{f}$, either to 0 . This latter limit is not suitable for our purposes, since it means that no noise will be extracted from our signal. One of the main questions raised by the peeling algorithm is thus to find an appropriate constant $F$ in (3) such that (i) The algorithm yields a convergence to a non trivial threshold $T_{f}>0$. (ii) $F$ is small enough, so that a sufficient part of the original signal is retrieved.

The previous attempts in this direction were simply (see [9]) to take $F=3 \sigma$ with experimental arguments; after the analysis performed in [15], this quantity was reduced to $F=F_{m}$, a quantity which is defined by

$$
F_{m}=\sqrt{\frac{3 \Gamma(1 / u)}{u}(u e)^{1 / u}}
$$

However, the latter bound has been obtained thanks to some rough estimates, and we have thus decided here to go one step further into this direction. Indeed, our first task will be to determine precisely, on a mathematical ground, a constant $F_{c}=F_{c}(u, \sigma)$ such that: if $F>F_{c}$, the algorithm yields a convergence, with high probability, to a strictly positive constant $T_{f}=T_{f}(\omega)$. In particular, we will see that our constant $F_{c}$ is always lower than $F_{m}$. Whenever $F<F_{c}$, we also show that $T_{k}$ converges to 0 with high probability (see Proposition 3.4).

(2) In the regime $F>F_{c}$, we determine that the optimal number of steps for the peeling algorithm is of order $\log (N)$, where we recall that $N$ is the total number of wavelet coefficients involved in the analysis. After this optimal number of steps, Theorem 3.1 quantifies also sharply the oscillations of $T_{f}$ with respect to a typical non-random value.

It is important to show that our theoretical results can really be applied to real data. We have thus decided first to compare the performances of our algorithm with other wavelet denoising procedures, on some classical benchmark signals proposed in [6]. It will be seen that our algorithm performs well with respect to other methods, independently of the value of the shape parameter in (5) and of the form of the benchmark signal.

A second step in our practical part of the study is the following: since the peeling algorithm has been introduced first in a medical context, we give an illustration of its performances on ECG type signals. More specifically, we shall consider a simulated ECG signal, and observe the denoising effect of our algorithm on a perturbed version of those electrocardiograms. As we shall see, the algorithm under analysis is a good compromise between denoising and preservation of the original signal. We also investigate the behavior of our method with non Gaussian noises, which is a possibility left open by our theoretical analysis.

Let us mention some open problems that have been left for a subsequent publication: first, let us recall that the so-called block thresholding has improved the behavior of 
the original thresholding algorithm in a certain number of situations (see e.g. [2] for a nice overview). It would be interesting to analyze the effect of this procedure in our peeling context. In relation to this problem, one should also care about some reasonable dependence structure among wavelet coefficients, beyond the independent case treated in this article. Finally, we have assumed in this paper that the parameters of the distributions $p_{\sigma, u}$ and $p_{\sigma_{w}}$ were known, which is typically not true in real world applications. One should thus be able to quantify the effect of parameter estimation on the whole denoising process.

Here is how our article is structured: we show how to compute optimal constants for the peeling algorithm at Section 2. Then the probabilistic analysis of the algorithm is carried out at Section 3. Finally, some numerical experiment on simulated and pseudo-real data are performed at Section 4.

\section{CRitical CONSTANTS FOR The PEELING AlgORIthm}

This section is devoted to the computation of an optimal constant $F$ in equation (3), ensuring a convergence of the threshold $T_{k}$ to a non trivial $T_{f}$, and still allowing to retrieve a maximal amount of approximate signal from our noisy input $z$.

Let us start this procedure by changing slightly the setting of the peeling algorithm. Indeed, it will be essential for our convergence theorems at Section 3, to be able to express the fixed point algorithm in terms of empirical processes. To this purpose, we resort to a simple change of variables by setting:

$$
U_{k}=T_{k}^{2}, \quad U_{f}=T_{f}^{2} \quad \text { and } \quad Y(q)=z(q)^{2}=(x(q)+w(q))^{2} .
$$

Note that $U_{0}=+\infty$ and that the decreasing sequence $\left\{U_{k} ; k \geq 0\right\}$ converges to $U_{f}$. Moreover, for any integer $k \geq 0$, since $T_{k+1}=f_{N}\left(T_{k}\right)$ with $f_{N}$ defined by (3), we have:

$$
U_{k+1}=g_{N, w}\left(U_{k}\right)
$$

where

$$
g_{N, w}(t)=f_{N}^{2}(t)=\frac{F^{2}}{N} \sum_{q \leq N} Y(q) \mathbf{1}_{\{Y(q)<t\}} .
$$

The random fixed point $U_{f}=U_{f}(\omega)$ is then solution of the random equation

$$
g_{N, w}(t)=t .
$$

Remark 2.1. Observe that we have emphasized the dependence of the function $g_{N, w}$ on the noise $w$. The reader should be aware that in the sequel of the paper, $w$ denotes the noisy part of our signal, while $\omega$ stands for the generic element of an underlying probability space $(\Omega, \mathcal{F}, \mathbb{P})$.

We wish to find the critical (minimal) $F$ which ensures $U_{f}>0$. First, we reduce the random fixed point equation (9) to a deterministic one. Then we focus on the fixed point study of this deterministic fixed point equation. 
2.1. Reduction to a deterministic problem. We shall identify here a natural deterministic problem related to equation (9). Indeed, for $t \in \mathbb{R}_{+}$, the law of large numbers asserts that

$$
\text { a.s. }-\lim _{N \rightarrow \infty} g_{N, w}(t)=g_{\sigma, u, \sigma_{w}}(t)=2 F^{2} \int_{0}^{\sqrt{t}} y^{2}\left[p_{\sigma, u} * p_{\sigma_{w}}\right](y) d y,
$$

where $p_{\sigma, u}$ and $p_{\sigma_{w}}$ are defined at Hypothesis 1.1. Moreover, letting $\sigma_{w} \rightarrow 0_{+}$, for any $t \in \mathbb{R}_{+}$,

$$
\lim _{\sigma_{w} \rightarrow 0} g_{\sigma, u, \sigma_{w}}(t)=g_{\sigma, u}(t)=2 F^{2} \int_{0}^{\sqrt{t}} y^{2} p_{\sigma, u}(y) d y=F^{2} \sigma^{2} \Gamma_{\text {inc }}\left(\left(\frac{\beta}{\sigma} \sqrt{t}\right)^{u}, 3 / u\right)
$$

where $\Gamma_{\text {inc }}$ is the incomplete Gamma function and $\beta$ is defined at Hypothesis 1.1.

Though both equations (10) and (11) are expressed as simple limits of functions, it is thus reasonable to think that $U_{f}$ (fixed point of $g_{N, w}$ ) will be close to a fixed point of $g_{\sigma, u}$. This is indeed the content of our Theorem 3.1 and Remark 3.2. Our first aim is thus to give some sharp conditions on the coefficient $F$ ensuring that the equation the deterministic fixed point equation

has at least one solution $t>0$.

$$
g_{\sigma, u}(t)=t
$$

2.2. Deterministic fixed point study. In this section, we are first interested in the solutions of the equation (12) and then in the fixed point study of $g_{\sigma, u, \sigma_{w}}$.

Observe that a trivial change of variables $y \mapsto \sigma^{2} y$ in the integral (11) yields that

$$
g_{\sigma, u}(t)=\sigma^{2} g_{1, u}\left(t / \sigma^{2}\right) .
$$

Hence to solve equation (12) for any $\sigma>0$ and $u>0$, it is sufficient to deal with the case $\sigma=1$, up to replace $t$ by $v=t / \sigma^{2}$. An elementary analysis of $g_{1, u}$, whose details are left to the reader, leads to the following lemma.

Lemma 2.2. Recall that $g_{1, u}$ and $\beta$ are defined by (11) and (5) with $u>0$ the shape of the distribution of each wavelet coefficient $x(q)$.

(1) Then, the function $g_{1, u}$ is increasing, convex on $\left[0, \beta^{-2} u^{-2 / u}\right]$, concave on the interval $\left[\beta^{-2} u^{-2 / u},+\infty\right)$ and such that $\lim _{t \rightarrow \infty} g_{1, u}(t)=F^{2}$.

(2) Moreover there exists a critical value $F_{c}$, depending only on $u$, such that the following assertions hold.

(a) If $F<F_{c}$, the only fixed point of $g_{1, u}$ is 0 .

(b) If $F=F_{c}, g_{1, u}$ has exactly two fixed points 0 and $t_{c}^{*}>\beta^{-2} u^{-2 / u}$.

(c) If $F>F_{c}, g_{1, u}$ has exactly three fixed points $0, \ell_{1}>0$ and $t^{*}>\max \left(\ell_{1}, \beta^{-2} u^{-2 / u}\right)$.

The content of the above lemma is well illustrated by Figure 1.

Let us turn now to the computation of the critical coefficient $F_{c}$ and the critical fixed point $t_{c}^{*}$. In fact, it is also easy to show that $\left(F_{c}, t_{c}^{*}\right)$ is the solution $(F, r)$ of the system

$$
\left\{\begin{array}{l}
g_{1, u}^{\prime}(r)=1 \\
g_{1, u}(r)=r
\end{array}\right.
$$




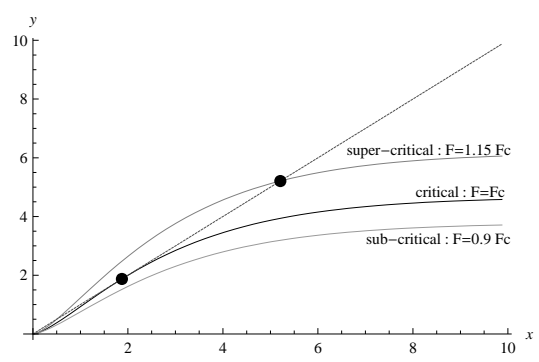

Figure 1. Curves corresponding to $g_{1, u}$ with $F \in\left\{0.9 F_{c}, F_{c}, 1.15 F_{c}\right\}$, with $u$ defined in Hypothesis 1.1 and $F_{c}$ in Lemma 2.2 .

where we recall that the coefficient $F$ enters into the definition of $g_{1, u}$. This system is equivalent to:

$$
\left\{\begin{aligned}
F^{2} \alpha \sqrt{r} e^{-(\beta \sqrt{r})^{u}}-1 & =0, \\
F^{2} \Gamma_{\text {inc }}\left((\beta \sqrt{r})^{u}, 3 / u\right)-r & =0
\end{aligned}\right.
$$

where it should be reminded that $\Gamma$ and $\Gamma_{\text {inc }}$ designate respectively Gamma and incomplete Gamma functions. The latter system can be solved with the Mathematica software, and the solutions for different $u$ are illustrated in Figure 2 and Table 1.

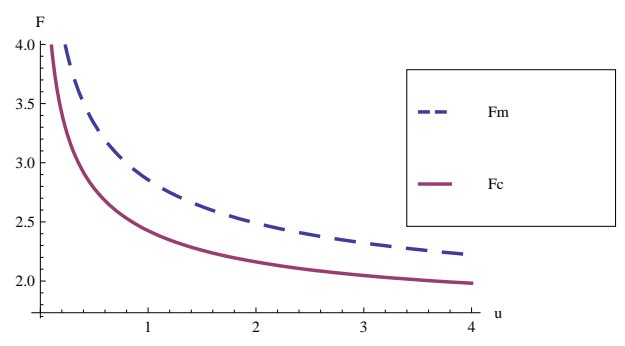

Figure 2. Evolution of $F_{m}$ and of critical $F_{c}$ (respectively defined in Equation (6) and Lemma 2.2) in function of the shape $u$ (defined in Hypothesis $1.1)$.

\begin{tabular}{c|cccccc}
$u$ & 0.1 & 0.5 & 1 & 2 & 3 & 4 \\
\hline$F_{c}$ & 4.0215 & 2.7830 & 2.42537 & 2.16169 & 2.0472 & 1.98181
\end{tabular}

TABLE 1. Critical constant $F_{c}$ for different shapes $u$. The shape $u$ is defined in Hypothesis 1.1 and the critical coefficient $F_{c}$ in Lemma 2.2.

In particular, as illustrated in Figure 2 it can be observed that $F_{c}<F_{m}$ where $F_{m}$ is the bound proposed by [15], and has been recalled at equation (6). 
We are now interested in the deterministic dynamic in presence of noise, that is in the sequence $\left\{u_{k}^{w} ; k \geq 0\right\}$ defined by

$$
\left\{\begin{aligned}
u_{0}^{w} & =+\infty \\
u_{k+1}^{w} & =g_{\sigma, u, \sigma_{w}}\left(u_{k}^{w}\right), \quad k \geq 0 .
\end{aligned}\right.
$$

with $g_{\sigma, u, \sigma_{w}}$ defined by (10). Indeed, the stochastic dynamic $\left\{U_{k} ; k \geq 0\right\}$ may converge to $U_{f}$ close to the limit of the deterministic sequence $\left\{u_{k}^{w} ; k \geq 0\right\}$, which is a fixed point of $g_{\sigma, u, w}$. We only focus on the case $F>F_{c}$, since we shall mainly analyze the performances of the peeling algorithm under this condition.

Proposition 2.3. For $\sigma>0, u>0$ and $\sigma_{w}>0$, let $g_{\sigma, u}$ and $g_{\sigma, u, \sigma_{w}}$ be defined by (11) and (10). Assume $F>F_{c}$ with $F_{c}$ introduced in Lemma 2.2. Recall that the fixed points of $g_{\sigma, u}$ are $0<\sigma^{2} \ell_{1}<\sigma^{2} t^{*}$, with $\ell_{1}$ and $t^{*}$ defined in Lemma 2.2. Then define the sequence $\left\{u_{k}^{w} ; k \geq 0\right\}$ by (15) and fix $M>F^{2}$. Then there exists three constants $\ell_{2} \in\left(0, t^{*}\right)$, $C \in(0, \infty), \tilde{M} \in(0,1)$, which depend neither on $\sigma$ nor on $\sigma_{w}$, such that for $\sigma_{w} / \sigma$ small enough, the following assertions hold.

(1) $\sup \left\{g_{\sigma, u, \sigma_{w}}(t) ; t \in \mathbb{R}_{+}\right\}<M \sigma^{2}$ and $g_{\sigma, u, \sigma_{w}}\left(\sigma^{2} \ell_{2}\right)>\sigma^{2} \ell_{2}$.

(2) $\sup \left\{g_{\sigma, u, \sigma_{w}}^{\prime}(t) ; t \in\left[\sigma^{2} \ell_{2}, \sigma^{2} M\right]\right\} \leq \tilde{M}<1$.

(3) The function $g_{\sigma, u, \sigma_{w}}$ has exactly one fixed point in $\left[\ell_{2} \sigma^{2}, \infty\right)$, called $t_{\sigma, w}^{*}$. Moreover $t_{\sigma, w}^{*} \in\left(\ell_{2} \sigma^{2}, M \sigma^{2}\right)$ and for any $k \geq 1$,

$$
\left|u_{k}^{w}-t_{\sigma, w}^{*}\right| \leq \sigma^{2} M \tilde{M}^{k-1} \text {. }
$$

Furthermore, the quantity $t_{\sigma, w}^{*}-\sigma^{2} t^{*}$ can be bounded as

$$
\left|t_{\sigma, w}^{*}-\sigma^{2} t^{*}\right| \leq C \sigma^{2}\left(\frac{\sigma_{w}}{\sigma}\right)^{\min (1, u)} .
$$

Proof. See Appendix, Section A.

Then, for $F>F_{c}$, the deterministic sequence $\left\{u_{k}^{w} ; k \geq 0\right\}$, given by (15) and obtained letting the number of wavelet coefficients $N$ goes to infinity in the peeling algorithm, converges to a positive value. Moreover, this limit is very close to the greater fixed point $\sigma^{2} t^{*}$ of $g_{\sigma, u}$ when the noise-to-signal ratio $\sigma_{w} / \sigma$ goes to 0 .

\section{Probabilistic AnAlysis of the Algorithm}

In this section, we are interested in the convergence for the peeling algorithm, that is in the random sequence $\left\{U_{k} ; k \geq 0\right\}$. Comparing this random sequence with the deterministic dynamic given by (15), we establish a concentration result on $\left\{U_{k} ; k \geq 0\right\}$.

In the supercritical case $F>F_{c}$, the following theorem states that the peeling algorithm converges to a fixed point which is close to a fixed point of the non-noisy deterministic dynamic governed by $g_{\sigma, u}$. The proof, which is given in Appendix B.2, hinges heavily on some empirical processes tools.

Theorem 3.1. Assume $F>F_{c}$ and that Hypothesis 1.1 is fulfilled. Let

$$
\eta_{u}=\min \left(\frac{u}{2}, 1\right)
$$

where $u$ is the shape parameter of the wavelet coefficients of the signal $x$. Let us also recall that $\sigma>0$ (respectively) is the variance of the wavelet coefficients of the signal $x$. Then, 
there exist some finite positive constant $c, C, A$ and $\widetilde{\gamma}$ such that if $\sigma_{w} / \sigma \leq c$ we have: for all $\alpha \in(0,1)$ and for all $N \in \mathbb{N}^{*}$, choosing $k=k(N):=[C \alpha \log (N)]+1$,

$$
\mathbb{P}\left(\left|U_{k}-t_{\sigma, w}^{*}\right| \geq N^{-\alpha / 2}\right) \leq A \mathrm{e}^{-\widetilde{\gamma} N^{\left[(1-\alpha) \eta_{u}\right] / 2}} .
$$

Remark 3.2. This theorem induces several informations about the convergence of our algorithm: ( $i)$ For a fixed number of wavelet coefficients $N$, the optimal number of iterations $n$ for the peeling algorithm is of order $\log (N)$. (ii) Once $n$ is fixed in this optimal way, $U_{k}$ is close to the fixed point $t_{\sigma, w}^{*}$ of $g_{\sigma, u, \sigma_{w}}$, the magnitude of $\left|U_{n}-t_{\sigma, w}^{*}\right|$ being of order $N^{-(1 / 2-\varepsilon)}$ for any $\varepsilon>0$. (iii) The deviations of $U_{k}$ from $t_{\sigma, w}^{*}$ are controlled exponentially in probability. (iv) Adding the information contained in Proposition 2.3, we obtain that $\left|t_{\sigma, w}^{*}-\sigma^{2} t^{*}\right|$ is of order $\sigma_{w} / \sigma$, where $t^{*}$ is the fixed point of function $g_{1, u}$ alluded to at Lemma A.1. Hence, if the ratio signal/noise is large, the iterated value $U_{k}$ of the peeling algorithm will be very close to $\sigma^{2} t^{*}$ when $k$ is of order $\log (N)$.

Remark 3.3. In Theorem 3.1, we have assumed a classical Gaussian structure for the noisy part $w$ of the signal. Notice however that we could have derived all our results with different distributions, up to a change on all the scaling factors in (18). This is the reason why we shall investigate the performances of the peeling algorithms with different shapes of noises at Section 4.

We finish this section by establishing that the choice of the constant $F_{c}$ for the peeling algorithm is optimal in the following sense: if one chooses a parameter $F<F_{c}$, then the threshold sequence converges to 0 with high probability. Specifically, we get the following result:

Proposition 3.4. Consider $F<F_{c}$ and assume that our signal z satisfies Hypothesis 1.1. Let $\alpha \in(0,1)$. Then, there exist some finite positive constants $c, C, A$ and $\widetilde{\gamma}$ such that if $\sigma_{w} / \sigma \leq c$, we have: for any $N \in \mathbb{N}^{*}$ and any integer $k \geq C \log (N)$,

$$
\mathbb{P}\left(U_{k} \geq N^{-\alpha / 2}\right) \leq A \mathrm{e}^{-\widetilde{\gamma} N^{(1-\alpha) \eta_{u} / 2}} .
$$

Remark 3.5. We have chosen here to investigate the case of a probability $\mathbb{P}\left(U_{k} \geq N^{-\alpha / 2}\right)$ and of a logarithmic number of iterations $k$, in order to be consistent with Theorem 3.1. However, in the simpler subcritical setting, one could have considered a number of iterations of order $N$, opening the door to a possible almost sure convergence of $U_{k}$ to 0 . We have not entered into those details for sake of conciseness. In the same spirit, we have not tried to solve the (much harder) problem of the behavior of our algorithm in the critical case $F=F_{c}$.

\section{Simulation Results}

The aim of this section is two-folded:

- We wish to illustrate by numerical simulations the analysis presented above, namely the peeling algorithm convergence.

- We will also evaluate the denoising performances of our method, and compare it to other thresholding algorithms.

These two goals and the respective simulation setups are detailed below. 


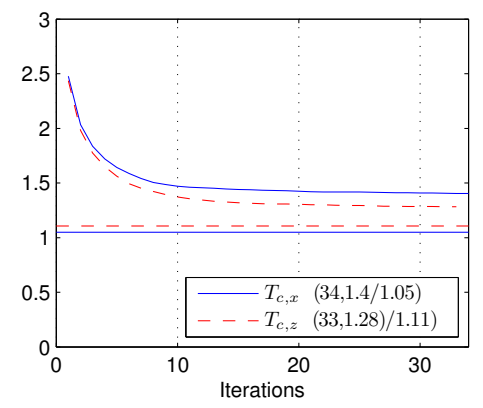

(A) $F_{c} / T_{c}$

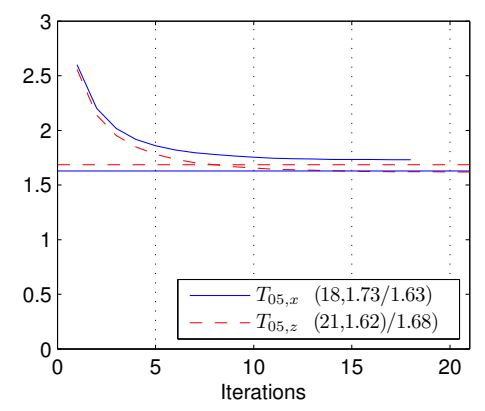

(B) $F_{05} / T_{05}$

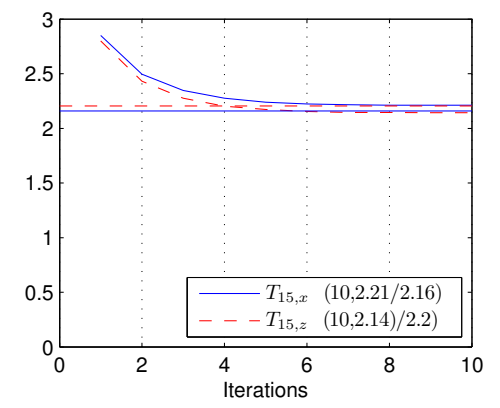

(C) $F_{15} / T_{15}$

Figure 3. Convergence speed for different versions of the peeling algorithms, based on the parameters $\left(u_{x}, \sigma_{x}\right)$ and $\left(u_{z}, \sigma_{z}\right)$. The constant lines correspond to the deterministic thresholds. The number of iterations until convergence and our final values for the thresholds, followed by the deterministic value given by Lemma A.1, are given in parenthesis. Recall that, for signals having $N=10000$ points, the convergence should occur after a number of iterations of order $\log (N)=9.21$.

4.1. Convergence issues. One of the assumptions made above was that the influence of the noise is limited (and obviously, controlled by its variance). Under this hypothesis, the peeling algorithm iterations should converge in about $\log (N)$ iterations to a deterministic threshold uniquely determined by the parameters of the signal probability law (assumed Generalized Gaussian). This convergence should take place for multiplicative constants $F$ greater than the critical value $F_{c}$.

The following simulation illustrates this convergence: we have generated the wavelet coefficients of an original signal $x$ according to a Generalized Gaussians (GG) law with zero mean, unitary standard deviation $\left(\sigma_{x}=1\right)$ and a shape factor $u_{x}=1$ (Laplace distribution). A Gaussian noise $w$ was then added with a signal to noise ratios $\mathrm{SNR}=5$. The length of the considered signals was $N=10000$ (namely $\log (N)=9.21$ ). With this simulated noisy signal in hand, we have performed the following steps:

(i) According to the real value of $u_{x}$, we have computed the critical constant $F_{c}$ solution of the Equations (14). We have also displayed two other values for the multiplicative constant $F$, both greater than $F_{c}$ (namely $F_{05}=1.05 F_{c}$ and $F_{15}=1.15 F_{c}$ ). We denote by $T_{c, x}, T_{05, x}$ and $T_{15, x}$ the thresholds corresponding to $F_{c}, F_{05}$ and $F_{15}$ respectively.

(ii) We have also pretended to know the observation $z=x+w$ only, which was assumed to follow a GG law. We have then estimated $u_{z}$ and $\sigma_{z}$ by means of classical moments methods as described in [11]. Once $u_{z}$ and $\sigma_{z}$ were estimated, our procedure (i) has been performed again, replacing $u_{x}, \sigma_{x}$ by $u_{z}, \sigma_{z}$ (the final threshold being obtained by Lemma A.1 and Proposition 2.3). We denote by $T_{c, z}, T_{05, z}$ and $T_{15, z}$ the thresholds corresponding to $F_{c}, F_{05}$ and $F_{15}$ respectively.

The results of steps (i) and (ii) have been compared to the deterministic thresholds obtained using the constants in Lemma A.1. The experiment, which exhibits a reasonable convergence of the iterated thresholds to the deterministic ones, is summarized in Figure 3. 

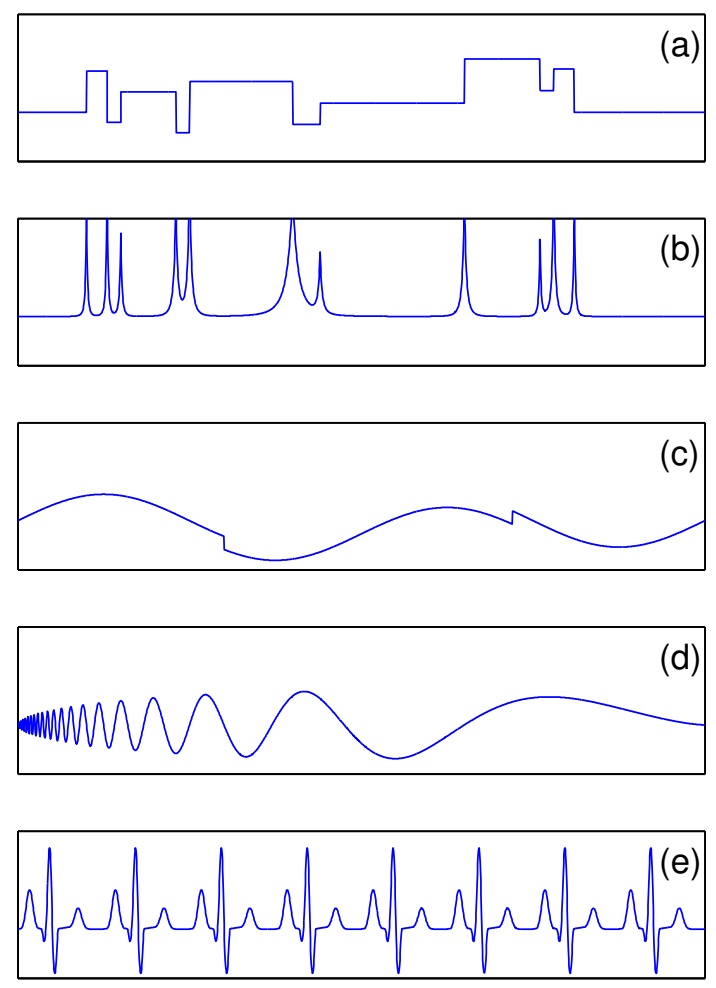

Figure 4. Clean test signals, from top to bottom: Blocks, Bumps, HeaviSine, Doppler, ECG

Similar results, not presented here, were obtained for other GG signals (varying $u_{x}$ ) and noises (not only Gaussian, but Generalized Gaussian), for relatively high SNRs (10 to 5). For lowed SNR, as expected the performances degrade: the peeling algorithms continue to converge in $\log (N)$ iterations, but the final value of the threshold becomes more significantly different from the deterministic value.

4.2. Denoising performances. The previous subsection aimed at shedding light on the convergence properties of the peeling algorithm. According to the presented analysis and the simulation results, the deterministic threshold can be used instead of the iterative algorithm. We now wish to test this algorithm in terms of denoising performances on an empirical basis, i.e., on benchmark and on realistic signals (the four classical signals proposed in [6] plus an ECG-like signal - recall that the peeling algorithms were proposed and mainly used in bio-medical applications $[4,9,16])$. The signals are displayed at Figure 4.

In real situations, one does not have access to the distribution of the underlying clean signal $x$ and to its parameters. Therefore our simulation assumes that the noisy signal $z=$ $x+w$ is not very far from $x$, which means that we can use estimated $u_{z}$ and $\sigma_{z}$ to compute the critical constant $F_{c}$. We denote by $T_{05, z}$ and $T_{15, z}$ the thresholds corresponding to $F_{05}$ and $F_{15}$ respectively.

Several simulations were made, using different types of white noise: Laplacian $u_{w}=1$, Gaussian $u_{w}=2$ and quasi-uniform $u_{w}=20$ (noted further on by $\sim$ Uniform) and SNRs $(10,5,2$ and 1$)$. 
Signals having $N=2048$ points were considered, the algorithm performance being evaluated using the SNR obtained after denoising. We considered here a level 5 decomposition and classical symlets with 8 null moments sym8.

We compare two traditional wavelet denoising procedures, namely Universal and SURE shrinkage (see [6]) with two of the peeling algorithms described above. Soft thresholding was implemented for all algorithms. The noise being white (namely, i.i.d coefficients with equal variance $\sigma_{w}^{2}$ for all scales), we did not considered scale-by-scale implementations for the compared algorithms (except for SURE, originally proposed with different thresholds by scale; note that peeling algorithms can also be implemented scale-by-scale).

To ease the comparisons, we present the results in a graphical manner in figure 5 .

A first analysis of these results indicates that, as expected, SURE thresholding is the best option for Gaussian noise, regardless of the SNR. The Heavisine signal is a particular case: indeed, aggressive high thresholds such as Universal and $T_{15, z}$ seem to work better, except when the noise is very small $(\mathrm{SNR}=10)$. This situation is even clearer for $\sim$ Uniform noise, when Heavisine makes no exception. It is interesting to note that $T_{15, z}$ thresholding performs generally better than Universal and it can constitute an alternative if the main goal is a quasi-complete noise elimination and a visually clean signal (recall that Universal thresholding is also known as VisuShrink because of its almost noise-free results).

On the other hand, the performances are quite different for Laplacian noise, when SURE thresholding performs very poorly. In this case, $T_{05, z}$ is the best option for rather lowpower noises, except for the Heavisine and Doppler benchmarks, when the high thresholds as Universal and $T_{15, z}$ become more performant (as for low SNR).

To conclude, the $T_{05, z}$ threshold derived from the minimal denoising peeling algorithm from [15] seems to be a good choice for heavy-tailed noises and high to moderate signal to noise ratios. On the contrary, for Gaussian and supra-Gaussian noises, SURE remains the best option for the considered model (i.e., independent wavelet coefficients, excluding thus block-thresholding algorithms like $[18,19])$. Notice however that our $T_{15, z}$ threshold performs similarly to SURE in this context, while preserving better the visual aspect of the signal. Furthermore, these conclusions could be challenged by scale-by-scale implementations of the $T_{c}$-type thresholds.

We finish this implementation section by presenting two examples of denoising of Blocks and ECG signals in Figure 6, which confirm the aforementioned considerations. Twodimensional versions of the tested algorithms have also been applied on real benchmark images (Lena, House, Barbara, Peppers), with similar performances to those obtained for the 1-D signals. They are not presented here for sake of conciseness.

\section{Appendix A. Proof of Proposition 2.3}

A.1. Preliminaries. This section gives the main tools to prove Proposition 2.3. The first lemma is interested in some useful properties of $g_{1, u}$, which is defined by (11), and in the associated deterministic dynamic.

Lemma A.1. Assume $F>F_{c}$. Let $g_{1, u}: \mathbb{R}_{+} \rightarrow \mathbb{R}_{+}$be defined by (11), where $u$ is the shape parameter given in Hypothesis 1.1. Let $\ell_{1}<t^{*}$ be the two positive fixed points of $g_{1, u}$ as defined in Lemma 2.2. 


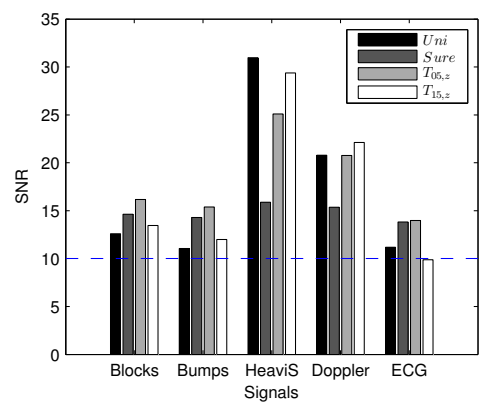

(A) Laplacian $/ \mathrm{SNR}=10$

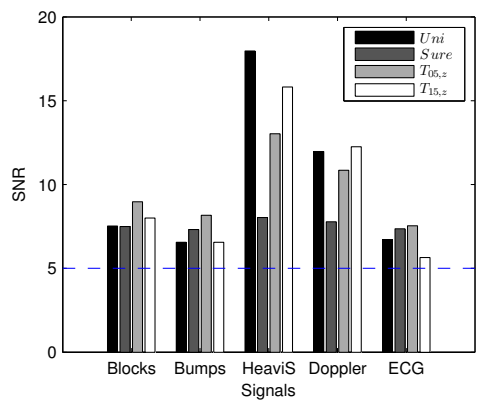

(D) Laplacian $/ \mathrm{SNR}=5$

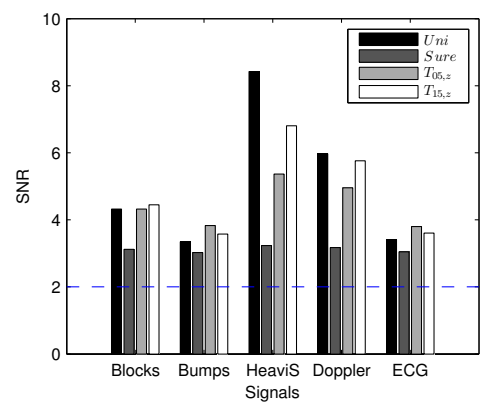

(G) Laplacian $/ \mathrm{SNR}=2$

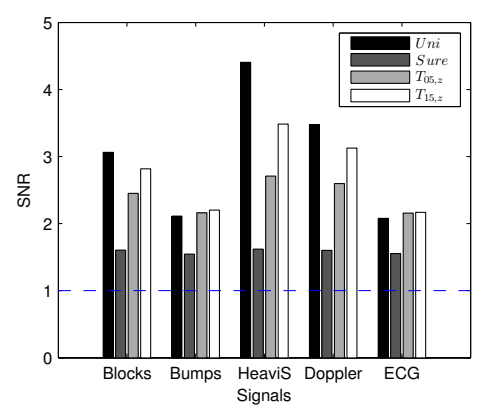

(J) Laplacian/SNR=1

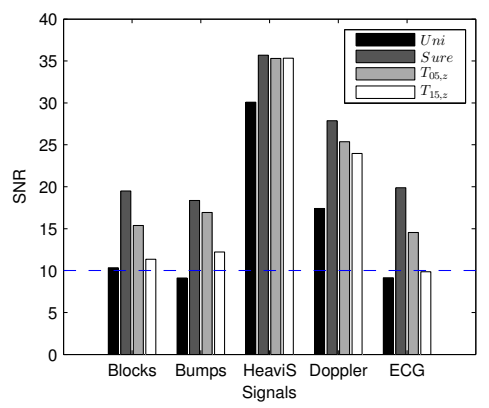

(B) Gaussian $/ \mathrm{SNR}=10$

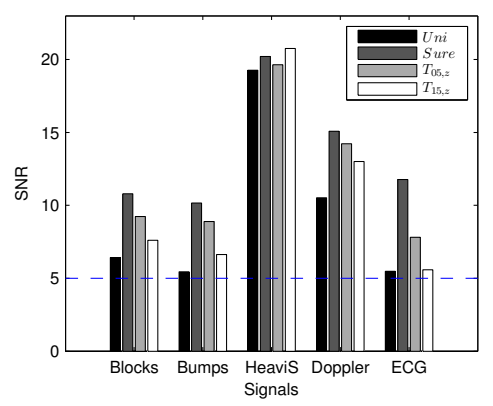

(E) Gaussian $/ \mathrm{SNR}=5$

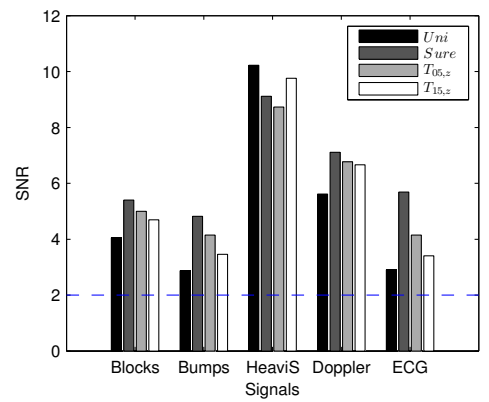

(H) Gaussian/SNR $=2$

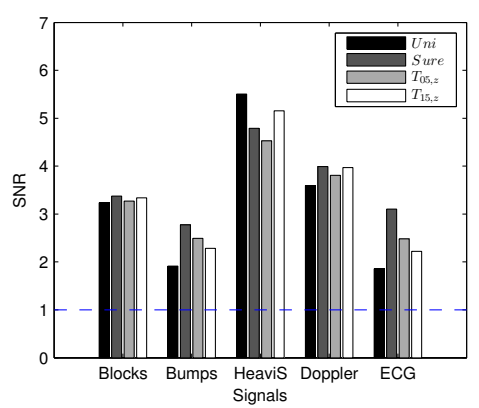

(K) Gaussian $/ \mathrm{SNR}=1$

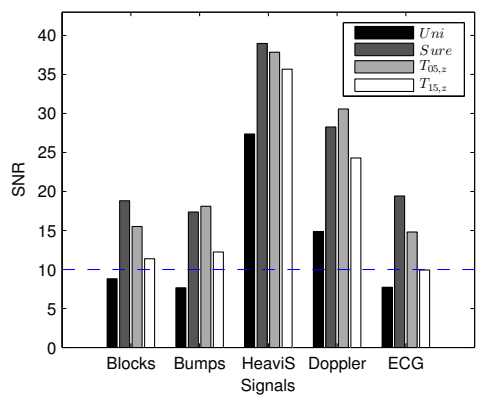

(C) $\sim$ Uniform $/ \mathrm{SNR}=10$

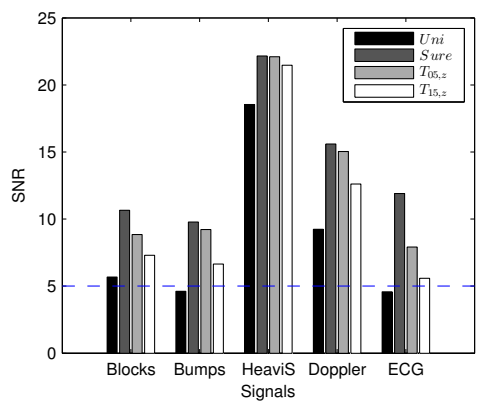

(F) $\sim$ Uniform $/ \mathrm{SNR}=5$

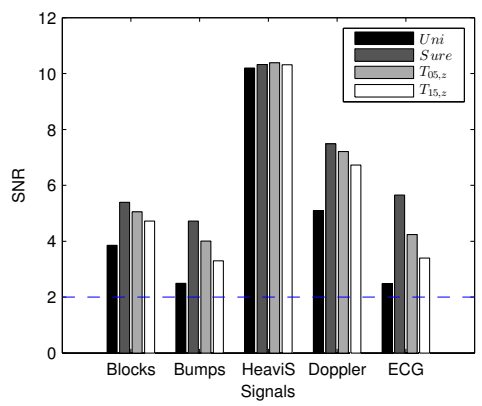

(I) $\sim$ Uniform $/ \mathrm{SNR}=2$

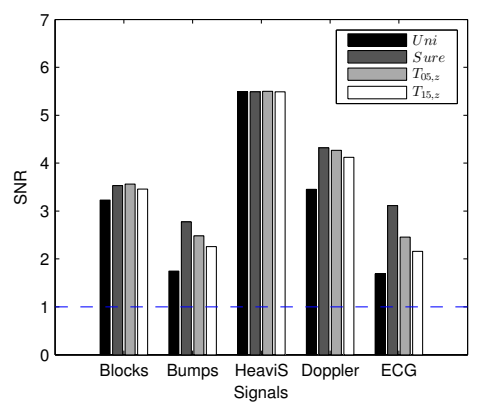

(L) $\sim$ Uniform $/ \mathrm{SNR}=1$

FiguRE 5. Denoising results comparisons between 4 algorithms on 5 signals. We considered Laplacian, Gaussian and $\sim$ Uniform noises, with SNR varying from 10 to 1 . The original $\mathrm{SNR} \sigma_{x} / \sigma_{w}$ before denoising is represented by the dotted line, while the bar heights represent the final SNR after denoising 

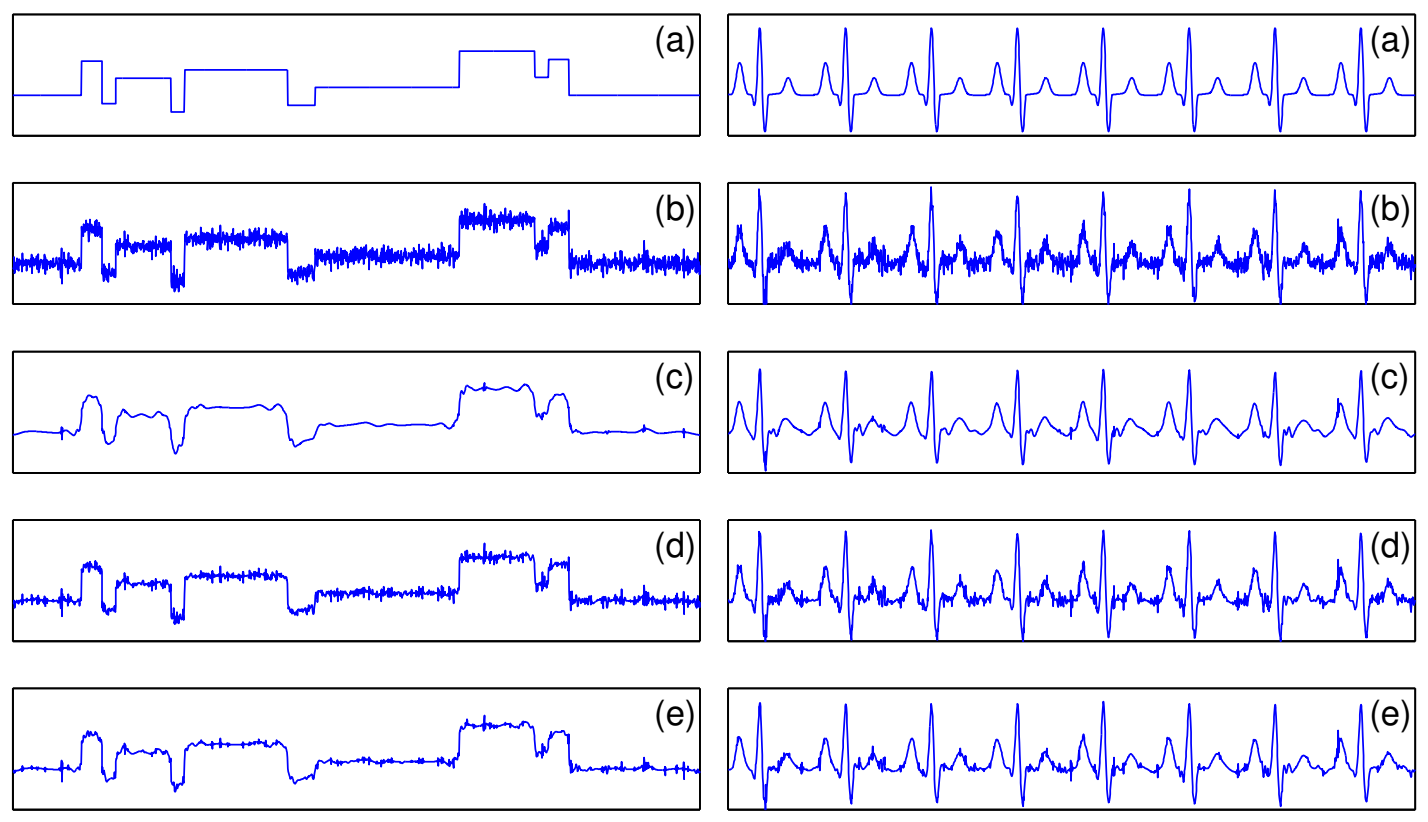

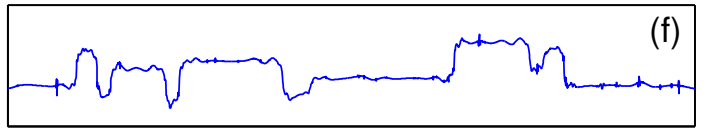

(A) Blocks

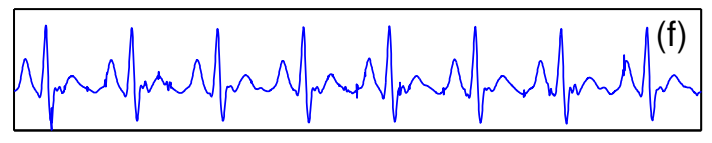

(в) ECG

Figure 6. Denoising example. From top to bottom: (a) clean signal; (b) noisy signal (Laplacian noise, $\mathrm{SNR}=3$ ); (c) Universal thresholding (Blocks: $\mathrm{SNR}=5.7$, ECG: $\mathrm{SNR}=4.8$ ); (d) SURE thresholding (Blocks: $\mathrm{SNR}=5.9$, ECG: $\mathrm{SNR}=5.4$ ); (e) $T_{05, z}$ thresholding (Blocks: SNR=6.8, ECG: $\mathrm{SNR}=5.9$ ); (f) $T_{15, z}$ thresholding (Blocks: $\mathrm{SNR}=6.4, \mathrm{ECG}: \mathrm{SNR}=5.2$ )

(1) Then there exists $\ell_{2} \in\left(\ell_{1}, t^{*}\right)$ such that $g_{1, u}\left(\ell_{2}\right)>\ell_{2}, g_{1, u}^{\prime}\left(\ell_{2}\right)<1$ and $g_{1, u}$ is concave on $\left[\ell_{2}, \infty\right)$.

(2) Define the deterministic sequence $\left\{u_{k} ; k \geq 0\right\}$ recursively by

$$
\left\{\begin{aligned}
u_{0} & =+\infty \\
u_{k+1} & =g_{1, u}\left(u_{k}\right), \quad k \geq 0 .
\end{aligned}\right.
$$

Then for $k \geq 1$,

$$
\left|u_{k}-t^{*}\right| \leq M_{t^{*}}^{k-1}\left(F^{2}-t^{*}\right)
$$

where $M_{t^{*}}=g_{1, u}^{\prime}\left(t^{*}\right) \in(0,1)$.

Proof. The first assertion is easily deduced from the variations of $t \mapsto d_{1, u}(t)=g_{1, u}(t)-t$, and its proof is left to the reader. Let us now prove the second assertion. According to Lemma 2.2, $g_{1, u}$ is an increasing function and has exactly three fixed points: $0<\ell_{1}<t^{*}$. Then the sequence $\left\{u_{k} ; k \geq 0\right\}$, defined by (20), is decreasing and converges to $t^{*}$ as $k \rightarrow \infty$. Furthermore,

$$
\left|u_{k+1}-t^{*}\right|=u_{k+1}-t^{*}=g_{1, u}\left(u_{k}\right)-g_{1, u}\left(t^{*}\right) \leq M_{t^{*}}\left(u_{k}-t^{*}\right),
$$


with $M_{t^{*}}=\sup \left\{\left|g_{1, u}^{\prime}(t)\right| ; t \geq t^{*}\right\}$. Since $g_{1, u}$ is increasing and concave on $\left[\ell_{2},+\infty\right)$ with $\ell_{2}<t^{*}$ and $g_{1, u^{\prime}}\left(\ell_{2}\right)<1$,

$$
M_{t^{*}}=g_{1, u}^{\prime}\left(t^{*}\right) \in(0,1) .
$$

Then, Assertion (2) follows by a trivial induction procedure. The proof of Lemma A.1 is then complete.

The following lemma compares the functions $g_{\sigma, u, \sigma_{w}}$ and $g_{\sigma, u}$ defined by (10) and (11) respectively.

Lemma A.2. Assume $F>F_{c}$. For $u>0, \sigma>0$ and $\sigma_{w}>0$, let $g_{\sigma, u}$ and $g_{\sigma, u, \sigma_{w}}$ be defined by (11) and (10) with $p_{\sigma, u}$ and $p_{\sigma_{w}}$ introduced in Hypothesis 1.1. Let $\ell_{1}<t^{*}$ be the two positive fixed points of $g_{1, u}$ as defined in Lemma 2.2. Then, there exists $C:=C(u) \in$ $(0, \infty)$ a constant which does not depend on $\left(\sigma, \sigma_{w}, F\right)$ such that for any $t \in \mathbb{R}_{+}$we have

$$
\left|g_{\sigma, u, w}^{\prime}(t)-g_{\sigma, u}^{\prime}(t)\right| \leq \frac{C F^{2} \sqrt{t}}{\sigma}\left(\frac{\sigma_{w}}{\sigma}\right)^{\min (1, u)}
$$

and

$$
\left|g_{\sigma, u, \sigma_{w}}(t)-g_{\sigma, u}(t)\right| \leq \frac{C F^{2} t^{3 / 2}}{\sigma}\left(\frac{\sigma_{w}}{\sigma}\right)^{\min (1, u)} .
$$

In particular, $g_{\sigma, u, \sigma_{w}} \rightarrow g_{\sigma, u}$ and $g_{\sigma, u, w}^{\prime} \rightarrow g_{\sigma, u}^{\prime}$ uniformly on every compact set of $\mathbb{R}^{+}$, as $\sigma_{w}$ goes to 0 .

Proof. Since (23) is a direct consequence of (22), we only prove (22). By definition of $g_{\sigma, u, \sigma_{w}}$ and $g_{\sigma, u}$, for any $t \in \mathbb{R}_{+}$,

$$
\left|g_{\sigma, u, \sigma_{w}}^{\prime}(t)-g_{\sigma, u}^{\prime}(t)\right|=F^{2} \sqrt{t}\left|p_{\sigma, u} * p_{\sigma w}(\sqrt{t})-p_{\sigma, u}(\sqrt{t})\right| .
$$

Notice that for all $y \in \mathbb{R}_{+}$

$$
p_{\sigma, u} * p_{\sigma_{w}}(y)-p_{\sigma, u}(y)=\int_{\mathbb{R}}\left(p_{\sigma, u}(r)-p_{\sigma, u}(y)\right) p_{\sigma_{w}}(y-r) d r .
$$

It can be readily checked that

$$
\forall t \in \mathbb{R}, p_{\sigma, u}(t)=\frac{1}{\sigma} p_{1, u}\left(\frac{t}{\sigma}\right)=\frac{\alpha}{\sigma} \mathrm{e}^{-\left|\frac{\beta t}{\sigma}\right|^{u}}
$$

Let us first assume $u \geq 1$. Then $t \mapsto p_{1, u}(t)$ is $\mathcal{C}^{1}$ on $\mathbb{R}$ and its derivate $p_{1, u}^{\prime}$ is bounded on $\mathbb{R}$. In this case,

$$
\left|p_{\sigma, u}(r)-p_{\sigma, u}(y)\right| \leq \frac{|r-y|\left\|p_{1, u}^{\prime}\right\|_{\infty}}{\sigma^{2}} .
$$

Assume now that $u \in(0,1]$. Then by the Mean Value Theorem applied to the exponential map,

$$
\left|p_{\sigma, u}(r)-p_{\sigma, u}(y)\right| \leq\left.\frac{\alpha \beta^{u}}{\sigma^{1+u}}|| r\right|^{u}-|y|^{u} \mid .
$$

Since for any $\gamma \in(0,1)$ and $0 \leq b \leq a, a^{\gamma}-b^{\gamma} \leq|a-b|^{\gamma}$, one checks that

$$
\left|p_{\sigma, u}(r)-p_{\sigma, u}(y)\right| \leq \frac{\alpha \beta^{u}}{\sigma^{1+u}}|r-y|^{u} .
$$


Plugging (26) or (27) in (25), we now get the existence of a finite positive constant $c:=c(u)$ which only depends on $u$ such that

$$
\left|p_{\sigma, u} * p_{\sigma_{w}}(y)-p_{\sigma, u}(y)\right| \leq \frac{c}{\sigma^{1+\min (1, u)}} \int_{\mathbb{R}}|v|^{\min (1, u)} p_{\sigma_{w}}(v) d v
$$

Since $p_{\sigma_{w}}$ is the density of a centered Gaussian variable of variance $\sigma_{w}$,

$$
\left|p_{\sigma, u} * p_{\sigma_{w}}(y)-p_{\sigma, u}(y)\right| \leq c \mathbb{E}\left(|W|^{\min (1, u)}\right)\left(\frac{\sigma_{w}}{\sigma}\right)^{\min (1, u)},
$$

with $W$ a standard Gaussian variable. This inequality and equation (24) lead to (22) setting $C=c \mathbb{E}\left(|W|^{\min (1, u)}\right)$, which concludes the proof.

A.2. Proof of Proposition 2.3. This section is devoted to the proof of Proposition 2.3. In this proof, $c$ and $C$ denote two unspecified positive and finite constant which may not be the same in each occurrence and depend neither on the standard deviation $\sigma$ of the signal $x$ nor on the standard deviation $\sigma_{w}$ of the noise $w$. Let us recall that $g_{\sigma, u, \sigma_{w}}$ is defined by (10).

(1) First observe that

$$
\forall t \in \mathbb{R}_{+}, g_{\sigma, u, \sigma_{w}}(t) \leq F^{2} \int_{0}^{+\infty} y^{2} p_{\sigma, u} * p_{\sigma_{w}}(y) d y=F^{2} \mathbb{E}\left(z(1)^{2}\right),
$$

owing to the fact that $p_{\sigma, u} * p_{\sigma_{w}}$ is the density of the wavelet coefficient $z(1)=$ $x(1)+w(1)$. Since the centered random variable $x(1)$ and $w(1)$ are independent, this leads to

$$
\forall t \in \mathbb{R}_{+}, g_{\sigma, u, \sigma_{w}}(t) \leq F^{2}\left(\sigma^{2}+\sigma_{w}^{2}\right) .
$$

Thanks to the relation $M>F^{2}$, there exists a finite positive constant $c_{1}:=$ $c_{1}(M, F)$ depending only on $M$ and $F$ so that, if $\sigma_{w} / \sigma \leq c_{1}, F^{2}\left(\sigma^{2}+\sigma_{w}^{2}\right)<M \sigma^{2}$ and henceforth,

$$
\sup \left\{g_{\sigma, u, \sigma_{w}}(t) ; t \in \mathbb{R}_{+}\right\}<M \sigma^{2} .
$$

Let $\ell_{2} \in\left(\ell_{1}, t^{*}\right)$ be defined by Lemma A.1. Note that $\ell_{2}$ only depends on $g_{1, u}$ and thus on both parameters $F$ and $u$. Since $t^{*}$ is a fixed point of the increasing function $g_{1, u}$, we get

$$
\ell_{2}<t^{*} \leq F^{2}=\lim _{t \rightarrow+\infty} g_{1, u}(t)<M .
$$

Hence, applying (23) and (13), we have :

$$
g_{\sigma, u, \sigma_{w}}\left(\sigma^{2} \ell_{2}\right) \geq \sigma^{2}\left(g_{1, u}\left(\ell_{2}\right)-C\left(\frac{\sigma_{w}}{\sigma}\right)^{\min (1, u)}\right)
$$

where $C:=C(M, F, u) \in(0,+\infty)$ does not depend on $\left(\sigma, \sigma_{w}\right)$. Since $g_{1, u}\left(\ell_{2}\right)>\ell_{2}$ by Lemma A.1, the previous equation leads to the existence of a constant $c:=$ $c(M, F, u) \in\left(0, c_{1}(M, F)\right]$, such that if $\sigma_{w} / \sigma \leq c$,

$$
g_{\sigma, u, \sigma_{w}}\left(\sigma^{2} \ell_{2}\right)>\sigma^{2} \ell_{2} .
$$

Then, the proof of Assertion (1) is complete. 
(2) According to (22) and (13),

$$
\forall t \in\left[\sigma^{2} \ell_{2}, \sigma^{2} M\right], g_{\sigma, u, w}^{\prime}(t) \leq g_{1, u}^{\prime}\left(\frac{t}{\sigma^{2}}\right)+C\left(\frac{\sigma_{w}}{\sigma}\right)^{\min (1, u)}
$$

where $C:=C(M, F, u) \in(0,+\infty)$. Thanks to Lemma A.1,

$$
\sup \left\{g_{1, u}^{\prime}(y), y \geq \ell_{2}\right\}=g_{1, u}^{\prime}\left(\ell_{2}\right)<1 .
$$

Then, choosing $c:=c(M, F, u)$ small enough, if $\sigma_{w} / \sigma \leq c$, we obtain

$$
\forall t \in\left[\sigma^{2} \ell_{2}, \sigma^{2} M\right], g_{\sigma, u, w}^{\prime}(t) \leq g_{1, u}^{\prime}\left(\ell_{2}\right)+C c_{2}^{\min (1, u)}:=\tilde{M}<1,
$$

which establishes Assertion (2).

(3) Let us now prove Assertion (3). Assume that $\sigma_{w} / \sigma \leq c$. By Assertions (1) and $(2), d_{\sigma, u, \sigma_{w}}: t \mapsto g_{\sigma, u, \sigma_{w}}(t)-t$ is a decreasing function on $\left[\sigma^{2} \ell_{2}, \sigma^{2} M\right]$ such that $d_{w}\left(\sigma^{2} \ell_{2}\right)>0$ and $d_{w}\left(\sigma^{2} M\right)<0$. Then, there exists an unique number $t_{\sigma, w}^{*} \in$ $\left(\sigma^{2} \ell_{2}, \sigma^{2} M\right)$ such that $g_{\sigma, u, \sigma_{w}}\left(t_{\sigma, w}^{*}\right)=t_{\sigma, w}^{*}$. Moreover, since $g_{\sigma, u, \sigma_{w}}$ takes its values in $\left[0, \sigma^{2} M\right), t_{\sigma, w}^{*}$ is the only fixed point for $g_{\sigma, u, \sigma_{w}}$ in $\left[\sigma^{2} \ell_{2}, \infty\right)$.

Consider now the sequence $\left\{u_{k}^{w} ; k \geq 0\right\}$ defined by equation (15). Since $g_{\sigma, u, \sigma_{w}}$ is an increasing function which admits as unique fixed point $t_{\sigma, w}^{*}$ in $\left[\sigma^{2} \ell_{2}, \infty\right)$, it is easily seen that $\left\{u_{k}^{w} ; k \geq 0\right\}$ is a decreasing sequence such that $\lim _{k \rightarrow \infty} u_{k}^{w}=t_{\sigma, w}^{*}$. Moreover, for any $k \geq 1, u_{k}^{w} \in\left[t_{\sigma, w}^{*}, F^{2}\left(\sigma^{2}+\sigma_{w}^{2}\right)\right] \subset\left[\sigma^{2} \ell_{2}, \sigma^{2} M\right]$. Then, using that $t_{\sigma, w}^{*} \in\left(\sigma^{2} \ell_{2}, \sigma^{2} M\right)$ is a fixed point and Equation (29), we get

$$
\left|u_{k+1}^{w}-t_{\sigma, w}^{*}\right| \leq \tilde{M}^{k}\left|u_{1}^{w}-t_{\sigma, w}^{*}\right|
$$

for any $k \geq 1$. We can now bound trivially $\left|u_{1}^{w}-t_{\sigma, w}^{*}\right|$ as follows:

$$
\left|u_{1}^{w}-t_{\sigma, w}^{*}\right|=u_{1}^{w}-t_{\sigma, w}^{*} \leq M \sigma^{2}
$$

so that we end up with

$$
\left|u_{k+1}^{w}-t_{\sigma, w}^{*}\right| \leq M \sigma^{2} \tilde{M}^{k}
$$

for any $k \geq 1$. This equation, which is equation (16) also holds for $k=0$.

Consider now the sequence $\left\{u_{k} ; k \geq 0\right\}$ defined by equation (20). Using (13), (23), (28) and the Mean Value Theorem, we get:

$$
\begin{aligned}
\left|u_{k+1}^{w}-\sigma^{2} u_{k+1}\right| & =\left|g_{\sigma, u, \sigma_{w}}\left(u_{k}^{w}\right)-\sigma^{2} g_{1, u}\left(u_{k}\right)\right| \\
& \leq\left|g_{\sigma, u, \sigma_{w}}\left(u_{k}^{w}\right)-g_{\sigma, u}\left(u_{k}^{w}\right)\right|+\sigma^{2}\left|g_{1, u}\left(u_{k}^{w} / \sigma^{2}\right)-g_{1, u}\left(u_{k}\right)\right| \\
& \leq C \sigma^{2}\left(\frac{\sigma_{w}}{\sigma}\right)^{\min (1, u)}+g_{1, u}^{\prime}\left(\ell_{2}\right)\left|u_{k}^{w}-\sigma^{2} u_{k}\right|
\end{aligned}
$$

since $u_{k}^{w} / \sigma^{2}, u_{k} \in\left[\ell_{2}, \infty\right)$. By iterating this procedure, with $C:=C(M, F, u)$ that may change in each occurrence, we get:

$$
\begin{aligned}
\left|u_{k+1}^{w}-\sigma^{2} u_{k+1}\right| & \leq C \sigma^{2}\left(\frac{\sigma_{w}}{\sigma}\right)^{\min (1, u)} \sum_{n=0}^{k-1} g_{1, u}^{\prime}\left(\ell_{2}\right)^{k}+g_{1, u}^{\prime}\left(\ell_{2}\right)^{k}\left|u_{1}^{w}-\sigma^{2} u_{1}\right| \\
& \leq C \sigma^{2}\left(\frac{\sigma_{w}}{\sigma}\right)^{\min (1, u)}+F^{2} \sigma_{w}^{2} g_{1, u}^{\prime}\left(\ell_{2}\right)^{k}
\end{aligned}
$$


since $g_{1, u}^{\prime}\left(\ell_{2}\right)<1, u_{1}^{w}=F^{2}\left(\sigma^{2}+\sigma_{w}^{2}\right)$ and $u_{1}=F^{2}$. Taking limits in the relation above as $k \rightarrow \infty$ we get (17), which ends the proof.

\section{Appendix B. Probabilistic analysis of the Algorithm}

B.1. Preliminaries: Comparison Noisy dynamics/ Deterministic dynamics. As mentioned at equation (8), the exact dynamics governing the sequence $\left\{U_{k} ; k \geq 0\right\}$ is of the form $U_{k+1}=g_{N, w}\left(U_{k}\right)$ with $g_{N, w}$ defined by (8). In order to compare this with the deterministic dynamics (15), let us recast this relation into:

$$
U_{k+1}=g_{\sigma, u, \sigma_{w}}\left(U_{k}\right)+\varepsilon_{k, N}, \quad \text { where } \quad \varepsilon_{k, N}=g_{N, w}\left(U_{k}\right)-g_{\sigma, u, \sigma_{w}}\left(U_{k}\right) .
$$

Notice that the errors $\varepsilon_{k, N}$ are far from being independent, which means that the relation above does not define a Markov chain. However, a fairly simple expression is available for $U_{k}$ :

Proposition B.1. Let $U_{k}$ be defined by (7), $g_{\sigma, u, \sigma_{w}}$ by (10) and $\varepsilon_{k, N}$ by (30). For $k \geq 0$, set $g_{\sigma, u, \sigma_{w}}^{\circ k}$ for the $k^{\text {th }}$ iteration of $g_{\sigma, u, \sigma_{w}}$. Then for $k \geq 0$, we have:

$$
U_{k}=g_{\sigma, u, \sigma_{w}}^{\circ k}\left(U_{0}\right)+R_{k}, \quad \text { with } \quad R_{k}=\sum_{p=0}^{k-1} \varepsilon_{p, N} \prod_{q=2}^{k-p} g_{\sigma, u, \sigma_{w}}^{\prime}\left(C_{p+q}\right),
$$

where the random variable $C_{j}(j \geq 2)$ is a certain real number within the interval $\left[g_{\sigma, u, \sigma_{w}}^{\circ(j-1)}\left(U_{0}\right) ; U_{j-1}\right]$. In the definition of $R_{k}$, we have also used the conventions $\prod_{q=2}^{1} a_{q}=1$ and $R_{0}=0$.

Proof. It is easily seen inductively that $R_{0}=0, R_{1}=\varepsilon_{0, N}$ and for $k \geq 1$

$$
R_{k+1}=g_{\sigma, u, \sigma_{w}}^{\prime}\left(C_{k+1}\right) R_{k}+\varepsilon_{k, N} .
$$

Hence, by a backward induction, we obtain:

$$
R_{k}=\sum_{j=1}^{k} \varepsilon_{k-j, N} \prod_{l=0}^{j-2} g_{\sigma, u, \sigma_{w}}^{\prime}\left(C_{k-l}\right)=\sum_{p=0}^{k-1} \varepsilon_{p, N} \prod_{q=2}^{k-p} g_{\sigma, u, \sigma_{w}}^{\prime}\left(C_{p+q}\right),
$$

which ends the proof.

A useful property of the errors $\varepsilon_{p, N}$ is that they concentrate exponentially fast (in terms of $N$ ) around 0 . This can be quantified in the following:

Lemma B.2. Assume that our signal $z=x+w$ satisfies Hypothesis 1.1, and recall that $F$ is defined by equation (3). Set

$$
\eta_{u}=\min \left(\frac{u}{2}, 1\right) \quad \text { and } \quad \gamma_{u}=\frac{1}{2^{\max \left(2 \eta_{u}-1,0\right)} F^{2 \eta_{u}}} \min \left(\frac{\beta^{u}}{\sigma^{u}}, \frac{1}{2 \sigma_{w}^{2}}\right)
$$

where parameters $u, \sigma, \sigma_{w}$ and $\beta$ are defined at Hypothesis 1.1. Then for every $0<\gamma<\gamma_{u}$, there exists a finite positive constant $K>0$ such that for all $N \geq 1$, for all $p \geq 0$ and for all $\lambda \in\left[0, \gamma N^{\eta_{u} / 2}\right]$,

$$
\mathbb{E}\left[\mathrm{e}^{\lambda\left|\varepsilon_{p, N}\right|^{\eta_{u}}}\right] \leq K
$$

Moreover, for all $N \geq 1, p \geq 0$ and $l>0$,

$$
\mathbb{P}\left(\left|\varepsilon_{p, N}\right| \geq l\right) \leq K \mathrm{e}^{-\gamma l^{\eta_{u}} N^{\eta_{u} / 2}} .
$$


Proof. Recall that $\varepsilon_{p, N}$ is defined by:

$$
\varepsilon_{p, N}=g_{N, w}\left(U_{p}\right)-g_{\sigma, u, \sigma_{w}}\left(U_{p}\right)=\frac{1}{N} \sum_{q=1}^{N}\left(F^{2} Y(q) \mathbf{1}_{\left\{Y(q)<U_{p}\right\}}-g_{\sigma, u, \sigma_{w}}\left(U_{p}\right)\right),
$$

for a collection $\{Y(q) ; q \leq N\}$ of i.i.d random variables, where $Y(q)=z(q)^{2}$. Moreover, $z(q)=x(q)+w(q)$, with $x(q)$ a centered generalized Gaussian random variable with parameter $u>0$ (whose density is given by (5)) and $w(q) \sim \mathcal{N}\left(0, \sigma_{w}^{2}\right)$. For a fixed positive $t$, the fluctuations $g_{N, w}(t)-g_{\sigma, u, \sigma_{w}}(t)$ are easily controlled thanks to the classical central limit theorem or large deviations principle. The difficulty in our case arises from the fact that $U_{p}$ is itself a random variable, which rules out the possibility of applying those classical results. However, uniform central limit theorems and deviation inequalities have been thoroughly studied, and our result will be obtained by translating our problem in terms of empirical processes like in [17].

In order to express $\varepsilon_{p, N}$ in terms of empirical processes, consider $t \in[0, \infty]$ and define $h_{t}: \mathbb{R}_{+} \rightarrow \mathbb{R}_{+}$by $h_{t}(v)=F^{2} v \mathbf{1}_{\{v<t\}}$. Next, for $f: \mathbb{R}_{+} \rightarrow \mathbb{R}$, set

$$
\mathbb{G}_{N} f=\frac{1}{\sqrt{N}} \sum_{q=1}^{N}[f(Y(q))-\mathbb{E}[f(Y(q))]],
$$

and with these notations in mind, observe that

$$
\mathbb{G}_{N} h_{t}=\frac{1}{\sqrt{N}} \sum_{q=1}^{N}\left[h_{t}(Y(q))-g_{\sigma, u, \sigma_{w}}(t)\right] .
$$

It is now easily seen that

$$
\varepsilon_{p, N}=\frac{\mathbb{G}_{N} h_{U_{p}}}{\sqrt{N}}
$$

and the key to our result will be to get good control on $\mathbb{G}_{N} h_{t}$ in terms of $N$, uniformly in $t \in[0, \infty]$.

Let us consider the class of functions $\mathcal{G}=\left\{h_{t} ; t \in[0,+\infty]\right\}$. According to the terminology of [17], the uniform central limit theorems are obtained when $\mathcal{G}$ is a Donsker class of functions. A typical example of Donsker setting is provided by some VC classes (see [17, Section 2.6.2]). The VC classes can be briefly described as sets of functions whose subgraphs can only shatter a finite collection of points, with a finite maximal cardinality, in $\mathbb{R}^{2}$. For instance, the collections of indicators

$$
\mathcal{F}=\left\{\mathbf{1}_{[0, t)} ; t \in[0,+\infty]\right\} .
$$

is a VC class. Thanks to [17, Lemma 2.6.18], $\mathcal{G}$ is also a VC class since it can be written as

$$
\mathcal{G}=\mathcal{F} \cdot h=\{f h ; f \in \mathcal{F}\},
$$

where $h: \mathbb{R}_{+} \rightarrow \mathbb{R}_{+}$is defined by $h(v)=h_{\infty}(v)=F^{2} v$.

In order to state our concentration result, we still need to introduce the envelope $\overline{\mathcal{G}}$ of $\mathcal{G}$, which is a function $\overline{\mathcal{G}}: \mathbb{R}_{+} \rightarrow \mathbb{R}$ defined as

$$
\overline{\mathcal{G}}(v)=\sup \{f(v) ; f \in \mathcal{G}\}, v \in \mathbb{R}_{+} .
$$


Note that in our particular example of application, we simply have $\overline{\mathcal{G}}=h$. Let us also introduce the following notation:

$$
\mathcal{N}\left[\mathbb{G}_{N} ; \mathcal{G}, \lambda, m\right]:=\mathbb{E}^{*}\left[e^{\lambda \sup _{f \in \mathcal{G}}\left|\mathbb{G}_{N} f\right|^{m}}\right], \quad \text { and } \quad \mathcal{N}[h ; \lambda, m]:=\mathbb{E}\left[e^{\lambda|h(Y)|^{m}}\right],
$$

where $\mathbb{E}^{*}$ is the outer expectation (defined in [17] for measurability issues), and $Y$ can be decomposed as $Y=(X+W)^{2}$ for a centered generalized Gaussian random variable $X$ with parameter $u>0$ and an independent variable $W \sim \mathcal{N}\left(0, \sigma_{w}^{2}\right)$. In (34), we also assume $\lambda>0$ and $m \geq 0$.

Then, since $\mathcal{G}$ is a VC class with measurable envelope, $\mathcal{G}$ is a Donsker class and [17, Theorem 2.14.5 p. 244] leads to:

$$
\mathcal{N}\left[\mathbb{G}_{N} ; \mathcal{G}, \lambda, m\right] \leq c \mathcal{N}[h ; \lambda, m],
$$

with $c$ a finite positive constant which does not depend on $N, \lambda$ and $\mathcal{G}$. Furthermore, since $Y$ can be decomposed as $Y=(X+W)^{2}$ and invoking the elementary inequality $(a+b)^{p} \leq 2^{\max (p-1,0)}\left(a^{p}+b^{p}\right)$, valid for $a, b \geq 0$ and $p>0$, it is readily checked that

$$
\mathcal{N}[h ; \lambda, m]<\infty
$$

for $\lambda<\gamma_{u}$ with $\gamma_{u}$ defined at (31), and where $m=\eta_{u}:=\min \left(\frac{u}{2}, 1\right)$. Recalling now that $\varepsilon_{p, N}=N^{-1 / 2} \mathbb{G}_{N} h_{U_{p}}$, we have obtained:

$$
\mathbb{E}\left[\mathrm{e}^{\lambda\left|N^{1 / 2} \varepsilon_{p, N}\right|^{\eta_{u}}}\right] \leq \mathcal{N}\left[\mathbb{G}_{N} ; \mathcal{G}, \lambda, \eta_{u}\right] \leq c \mathcal{N}\left[h ; \gamma, \eta_{u}\right]=K<\infty
$$

for $\lambda \leq \gamma<\gamma_{u}$, which easily implies our claim (32).

Let $l>0$. Then,

$$
\mathbb{P}\left(\left|\varepsilon_{p, N}\right| \geq l\right)=\mathbb{P}\left(\mathrm{e}^{\gamma N^{\eta_{u} / 2}\left|\varepsilon_{p, N}\right|^{\eta_{u}}} \geq \mathrm{e}^{\gamma l^{\eta_{u}} N^{\eta_{u} / 2}}\right)
$$

The concentration property (33) is thus an easy consequence of (32) and Markov's inequality.

B.2. Proof of Theorem 3.1. Observe first that, owing to Proposition B.1 and inequality (16), we have

$$
\left|U_{k}-t_{\sigma, w}^{*}\right|=\left|g_{\sigma, u, \sigma_{w}}^{\circ k}\left(U_{0}\right)-t_{\sigma, w}^{*}+R_{k}\right|=\left|u_{k}^{w}-t_{\sigma, w}^{*}+R_{k}\right| \leq M \tilde{M}^{k-1} \sigma^{2}+\left|R_{k}\right|,
$$

for any $k \geq 1$. Let then $\hat{\delta}>0$ and let us fix $k \geq 1$ such that

$$
M \tilde{M}^{k-1} \sigma^{2} \leq \frac{\hat{\delta}}{2}
$$

i.e.

$$
k \geq 1+\log \left(\hat{\delta} /\left(2 M \sigma^{2}\right)\right) / \log (\tilde{M}) .
$$

Then it is readily checked that:

$$
\mathbb{P}\left(\left|U_{k}-t_{\sigma, w}^{*}\right| \geq \hat{\delta}\right) \leq \mathbb{P}\left(\left|R_{k}\right| \geq \frac{\hat{\delta}}{2}\right),
$$


and we will now bound the probability in the right hand side of this inequality. To this purpose, let us introduce a little more notation: recall that $\ell_{2}$ has been defined at Lemma A.1 and for $n \geq 1$, let $\Omega_{n}$ be the set defined by

$$
\Omega_{n}=\left\{\omega \in \Omega ; \inf \left\{j \geq 0 / U_{j}(\omega) \leq \sigma^{2} \ell_{2}\right\}=n\right\}
$$

and set also

$$
\widetilde{\Omega}_{k}=\bigcup_{n=1}^{k} \Omega_{n} \cup\left\{U_{1}>M \sigma^{2}\right\}
$$

Then we can decompose (37) into:

$$
\mathbb{P}\left(\left|U_{k}-t_{\sigma, w}^{*}\right| \geq \hat{\delta}\right) \leq \mathbb{P}\left(\widetilde{\Omega}_{k}\right)+\mathbb{P}\left(\widetilde{\Omega}_{k}^{c} \cap\left\{\left|R_{k}\right| \geq \frac{\hat{\delta}}{2}\right\}\right) .
$$

We will now control these two terms separately.

Step 1: Upper bound for $\mathbb{P}\left(\widetilde{\Omega}_{k}\right)$. Let us fix $n \geq 1$ and first study $\mathbb{P}\left(\Omega_{n}\right)$. To this purpose, observe first that

$$
\Omega_{n} \subset\left\{U_{n} \leq \sigma^{2} \ell_{2}<U_{n-1}\right\} .
$$

Hence, since $U_{n}=g_{N, w}\left(U_{n-1}\right)$ and invoking that $g_{\sigma, u, \sigma_{w}}$ is an increasing function, the following relation holds true on $\Omega_{n}$ :

$$
g_{N, w}\left(U_{n-1}\right)=U_{n} \leq \sigma^{2} \ell_{2} \quad \text { and } \quad g_{\sigma, u, \sigma_{w}}\left(\sigma^{2} \ell_{2}\right)<g_{\sigma, u, \sigma_{w}}\left(U_{n-1}\right) .
$$

We have thus proved that

$$
\Omega_{n} \subset\left\{g_{N, w}\left(U_{n-1}\right)-g_{\sigma, u, \sigma_{w}}\left(U_{n-1}\right) \leq \sigma^{2} \ell_{2}-g_{\sigma, u, \sigma_{w}}\left(\sigma^{2} \ell_{2}\right)\right\},
$$

where, by Assertion (1) of Proposition 2.3, $\sigma^{2} \ell_{2}-g_{\sigma, u, \sigma_{w}}\left(\sigma^{2} \ell_{2}\right)=$ : $-L_{1}<0$. Since $g_{N, w}\left(U_{n-1}\right)-g_{\sigma, u, \sigma_{w}}\left(U_{n-1}\right)=\varepsilon_{n-1, N}$ by definition, we end up with:

$$
\mathbb{P}\left(\Omega_{n}\right) \leq \mathbb{P}\left(\left|\varepsilon_{n-1, N}\right| \geq L_{1}\right) \text {. }
$$

Moreover,

$$
\mathbb{P}\left(U_{1}>M \sigma^{2}\right) \leq \mathbb{P}\left(\left|\varepsilon_{0, N}\right|>L_{2}\right)
$$

with $L_{2}=M \sigma^{2}-g_{\sigma, u, \sigma_{w}}(+\infty)>0$ by Assertion (1) of Proposition 2.3.

A direct application of Lemma B.2 yields now the existence of $\gamma, K \in(0, \infty)$ such that for all $n \geq 1$ and all $N \geq 1$

$$
\mathbb{P}\left(\Omega_{n}\right) \leq K \mathrm{e}^{-\gamma L_{1}^{\eta_{u}} N^{\eta_{u} / 2}} \quad \text { and } \quad \mathbb{P}\left(U_{1}>M \sigma^{2}\right) \leq K \mathrm{e}^{-\gamma L_{2}^{\eta_{u}} N^{\eta_{u} / 2}}
$$

with $\eta_{u}=\min \left(\frac{u}{2}, 1\right)$. Hence

$$
\mathbb{P}\left(\widetilde{\Omega}_{k}\right) \leq \sum_{n=1}^{k} \mathbb{P}\left(\Omega_{n}\right)+\mathbb{P}\left(U_{1}>M \sigma^{2}\right) \leq(k+1) K \mathrm{e}^{-\gamma L^{\eta_{u}} N^{\eta_{u} / 2}}
$$

where $L:=\min \left(L_{1}, L_{2}\right)>0$.

Step 2: Upper bound for $\mathbb{P}\left(\widetilde{\Omega}_{k}^{c} \cap\left\{\left|R_{k}\right| \geq \frac{\hat{\delta}}{2}\right\}\right)$. We have constructed the set $\widetilde{\Omega}_{k}$ so that, for all $2 \leq p \leq k+1$, the random variables $C_{p}$ introduced at Proposition B.1 satisfy $0 \leq g_{\sigma, u, \sigma_{w}}^{\prime}\left(C_{p}\right) \leq \rho:=\tilde{M}_{\ell_{2}}<1$ on $\widetilde{\Omega}_{k}^{c}$. Thus

$$
\mathbb{P}\left(\widetilde{\Omega}_{k}^{c} \cap\left\{\left|R_{k}\right| \geq \frac{\hat{\delta}}{2}\right\}\right) \leq \mathbb{P}\left(\sum_{p=0}^{k-1}\left|\varepsilon_{p, N}\right| \rho^{k-1-p} \geq \frac{\hat{\delta}}{2}\right) \leq \mathbb{P}\left(\sum_{p=0}^{k-1}\left|\varepsilon_{p, N}\right| \nu_{p} \geq L_{k, \hat{\delta}}\right)
$$


where we have set

$$
\nu_{p}=\frac{\rho^{k-1-p}(1-\rho)}{1-\rho^{k}}, \quad \text { and } \quad L_{k, \hat{\delta}}=\frac{\hat{\delta}(1-\rho)}{2\left(1-\rho^{k}\right)},
$$

so that $\left\{\nu_{p} ; 0 \leq p \leq k-1\right\}$ is a probability measure on $\{0, \ldots, k-1\}$.

We introduce now a convex non-decreasing function $a_{u}$ which only depends on the shape parameter $u$, and which behaves like $\exp \left(t^{\eta_{u}}\right)$ at infinity. Observe that, setting $s_{u}=\left(1 / \eta_{u}-1\right)^{1 / \eta_{u}}$, the function $t \mapsto \exp \left(t^{\eta_{u}}\right)$ is concave on $\left[0, s_{u}\right]$ and convex on $\left[s_{u},+\infty\right)$ Then, we consider the convex function $a_{u}$ defined by

$$
a_{u}(t)=\mathrm{e}^{t^{\eta_{u}}} \mathbf{1}_{\left[s_{u}, \infty\right)}(t)+\mathrm{e}^{s_{u}^{\eta_{u}}} \mathbf{1}_{\left[0, s_{u}\right)}(t) .
$$

Observe that if $u \geq 2, a_{u}$ is the exponential map.

Since $a_{u}$ is a non-decreasing function, for all $\lambda>0$, relation (40) implies that:

$$
\begin{aligned}
\mathbb{P}\left(\widetilde{\Omega}_{k}^{c} \cap\left\{\left|R_{k}\right| \geq \frac{\hat{\delta}}{2}\right\}\right) & \leq \mathbb{P}\left(a_{u}\left(\lambda \sum_{p=0}^{k-1}\left|\varepsilon_{p, N}\right| \nu_{p}\right) \geq a_{u}\left(\lambda L_{k, \hat{\delta}}\right)\right) \\
& \leq \frac{1}{a_{u}\left(\lambda L_{k, \hat{\delta}}\right)} \mathbb{E}\left[a_{u}\left(\lambda \sum_{p=0}^{k-1}\left|\varepsilon_{p, N}\right| \nu_{p}\right)\right],
\end{aligned}
$$

where we have invoked Markov's inequality for the second step. Hence, applying Jensen's inequality, for all $\lambda>0$, we obtain:

$$
\mathbb{P}\left(\widetilde{\Omega}_{k}^{c} \cap\left\{\left|R_{k}\right| \geq \frac{\hat{\delta}}{2}\right\}\right) \leq \frac{1}{a_{u}\left(\lambda L_{k, \hat{\delta}}\right)} \sum_{p=0}^{k-1} \nu_{p} \mathbb{E}\left(a_{u}\left(\lambda\left|\varepsilon_{p, N}\right|\right)\right)
$$

Furthermore, owing to the definition (41) of $a_{u}$,

$$
\mathbb{E}\left(a_{u}\left(\lambda\left|\varepsilon_{p, N}\right|\right)\right) \leq \mathbb{E}\left(\mathrm{e}^{\lambda^{\eta_{u}}\left|\varepsilon_{p, N}\right|^{\eta_{u}}}\right)+\mathrm{e}^{1 / \eta_{u}-1}
$$

for all $p \geq 0$, all $N \geq 1$ and all $\lambda>0$.

Then, applying Lemma B.2, we have:

$$
\mathbb{P}\left(\widetilde{\Omega}_{k}^{c} \cap\left\{\left|R_{k}\right| \geq \frac{\hat{\delta}}{2}\right\}\right) \leq \frac{K+\mathrm{e}^{1 / \eta_{u}-1}}{a_{u}\left(\lambda L_{k, \hat{\delta}}\right)}
$$

for any $\lambda \leq \gamma^{1 / \eta_{u}} N^{1 / 2}$ with $\gamma<\gamma_{u}$. Since $L_{k, \hat{\delta}} \geq(1-\rho) \hat{\delta} / 2$ and since $a_{u}$ is a non-decreasing function, by choosing $\lambda=\gamma^{1 / \eta_{u}} N^{1 / 2}$, we obtain:

$$
\mathbb{P}\left(\widetilde{\Omega}_{k}^{c} \cap\left\{\left|R_{k}\right| \geq \frac{\hat{\delta}}{2}\right\}\right) \leq \frac{K_{1}}{a_{u}\left(\gamma_{1} \hat{\delta} N^{1 / 2}\right)}
$$

with $\gamma_{1}=(1-\rho) \gamma^{1 / \eta_{u}} / 2>0$ and $K_{1}=K+\mathrm{e}^{1 / \eta_{u}-1}$.

Choose now $\hat{\delta}=N^{-\alpha / 2}$, with $\alpha<1$. Observe that for $N$ large enough, $\gamma_{1} \hat{\delta} N^{1 / 2}>s_{u}$ and thus $a_{u}\left(\gamma_{1} \hat{\delta} N^{1 / 2}\right)=\mathrm{e}^{\gamma_{1}^{\eta_{u}} N^{(1-\alpha) \eta_{u} / 2}}$. Hence, there exists a finite positive constant $K^{\prime}$ 
such that for all $N \geq 1$

$$
\mathbb{P}\left(\widetilde{\Omega}_{k}^{c} \cap\left\{\left|R_{k}\right| \geq \frac{1}{2 N^{\alpha / 2}}\right\}\right) \leq K^{\prime} \mathrm{e}^{-\widetilde{\gamma} N^{(1-\alpha) \eta_{u} / 2}}
$$

with $\widetilde{\gamma}=\gamma_{1}^{\eta_{u}}$.

Step 3: Conclusion. Putting together (38), (39) and (42), choosing $\hat{\delta}=N^{-\alpha / 2}$ with $\alpha<1$, we end up with:

$$
\mathbb{P}\left(\left|U_{k}-t_{\sigma, w}^{*}\right| \geq N^{-\alpha / 2}\right) \leq(k+1) K \mathrm{e}^{-\gamma L^{\eta_{u}} N^{\eta_{u} / 2}}+K^{\prime} \mathrm{e}^{-\widetilde{\gamma} N^{(1-\alpha) \eta_{u} / 2}},
$$

for any $k$ satisfying (36). Choose now $k=k(N):=[C \alpha \log (N)]+1$. If the following condition holds true:

$$
\lim _{N \rightarrow+\infty}\left(k+\frac{\alpha}{2} \log (N) / \log (\tilde{M})\right)=+\infty
$$

i.e. if $C>-1 /(2 \log (\tilde{M}))$, then for $N \geq N_{0}$ with $N_{0}$ large enough, (36) holds. We thus choose $C=-1 /(2 \log (\tilde{M}))+\eta$ with $\eta>0$. Then, for $N \geq N_{0}$ and $k=k(N):=$ $[C \alpha \log (N)]+1$, we have (43). Therefore, since $(1-\alpha) \eta_{u} / 2 \leq \eta_{u} / 2$ we have proved that there exists a positive finite constant $A$ such that for all $N \in \mathbb{N}^{*}$,

$$
\mathbb{P}\left(\left|U_{k}-t_{\sigma, w}^{*}\right| \geq N^{-\alpha / 2}\right) \leq A \mathrm{e}^{-\widetilde{\gamma} N^{(1-\alpha) \eta_{u} / 2}},
$$

which is the desired result.

B.3. Proof of Proposition 3.4. In the subcritical case, the following property holds true for the function $g_{1, u}$ defined by (13): there exists a constant $\kappa_{1} \in(0,1)$ such that, for all $t \geq 0,0 \leq g_{1, u}(t) \leq \kappa_{1} t$.

Let us now fix $\kappa_{2} \in\left(\kappa_{1}, 1\right)$ and $L \in(0, \infty)$. Then, by (13) and (23), for $\sigma_{w} / \sigma \leq c$ with $c$ small enough,

$$
\forall t \in[0, L], 0 \leq g_{\sigma, u, \sigma_{w}}(t) \leq \kappa_{2} t .
$$

Since $g_{\sigma, u, \sigma_{w}}$ is upper bounded by $2 F^{2} \sigma^{2}$ (for $\sigma_{w} / \sigma \leq c$ with $c$ small enough), choosing $L$ such that $\kappa_{2} L>2 F^{2} \sigma^{2}$, the previous equation holds on $[0, \infty)$. We thus have the following relation for the noisy dynamics of $U_{k}$ : for every $k \geq 2$,

$$
U_{k}=g_{\sigma, u, \sigma_{w}}\left(U_{k-1}\right)+\varepsilon_{k-1, N} \leq \kappa_{2} U_{k-1}+\varepsilon_{k-1, N} .
$$

Iterating this inequality, we have: for every $k \geq 2$,

$$
U_{k} \leq \kappa_{2}^{k-1} U_{1}+\sum_{j=1}^{k-1} \kappa_{2}^{j-1} \varepsilon_{k-j, N} .
$$

According to the fact that $U_{1}=F^{2}\left(\sigma^{2}+\sigma_{w}^{2}\right)+\varepsilon_{0, N}$, we end up with:

$$
U_{k} \leq \kappa_{2}^{k-1} F^{2}\left(\sigma^{2}+\sigma_{w}^{2}\right)+\sum_{j=1}^{k} \kappa_{2}^{j-1} \varepsilon_{k-j, N},
$$

a relation which is valid for any $k \geq 1$.

Consider now $\alpha<1$, assume that $\sigma_{w} / \sigma \leq c$ and choose $C>-\alpha /\left(2 \log \left(\kappa_{2}\right)\right)$. Then there exists $N_{0} \in \mathbb{N}^{*}$ such that for any integers $N \geq N_{0}$ and $k \geq C \log (N)$,

$$
\kappa_{2}^{k-1} F^{2}\left(\sigma^{2}+\sigma_{w}^{2}\right) \leq \kappa_{2}^{k-1} F^{2} \sigma^{2}\left(1+c^{2}\right) \leq \frac{N^{-\alpha / 2}}{2} .
$$


Hence, for any integers $N \geq N_{0}$ and $k \geq C \log (N)$, invoking (44), we have:

$$
\mathbb{P}\left(U_{k} \geq N^{-\alpha / 2}\right) \leq \mathbb{P}\left(\sum_{j=1}^{k} \kappa_{2}^{j-1} \varepsilon_{k-j, N} \geq \frac{N^{-\alpha / 2}}{2}\right)
$$

We are thus back to the setting of the proof of Theorem 3.1, Step 2. Along the same lines as in this proof (changing just the name of the constants there), the reader can now easily check inequality (19).

\section{REFERENCES}

[1] A. Antoniadis, J. Bigot, T. Sapatinas: Wavelet estimators in non-parametric regression: a comparative simulation study. Preprint, 2006.

[2] C. Chesneau: Wavelet block thresholding for samples with random design: a minimax approach under the $L^{p}$ risk. Electron. J. Stat. 1 (2007), 331-346.

[3] R. Coifman, D. Donoho: Translation invariant denoising. Wavelets and Statistics, ed. A. Antoniadis and G. Oppenheim, Springer Verlag, 125-150 (1995).

[4] R. Coifman, M. Wickerhauser: Adapted waveform de-noising for medical signals an images, IEEE Engineering in Medecine and Biology Magazine 14, no. 5, 578-586 (1995).

[5] I. Daubechies: Ten lectures on wavelets. Society for Industrial and Applied Mathematics (1992).

[6] D. Donoho, I. Johnstone: Ideal spatial adaptation via wavelet shrinkage. Biometrika 81, 425-455 (1994).

[7] D. Donoho, I. Johnstone, G. Kerkyacharian, D. Picard: Wavelet shrinkage: asymptopia? With discussion and a reply by the authors. J. Roy. Statist. Soc. Ser. B 57, no. 2, 301-369 (1995).

[8] E. Gin, R. Nickl: Uniform limit theorems for wavelet density estimators. Ann. Probab. 37, no. 4, 1605-1646 (2009).

[9] L. Hadjileontiadis, S. Panas: Separation of discontinuous adventitious sounds from vesicular sounds using a wavelet based filter. IEEE Transactions on Biomedical Engineering 44, no. 12, 1269-1281 (1997).

[10] K. Kokkinakis, A. Nandi: Exponent parameter estimation for generalized Gaussian probability density functions with application to speech modeling. Signal Processing 85, 1852-1858 (2005).

[11] S. Mallat: A Theory for Multiresolution Signal Decomposition: The Wavelet Representation. IEEE Transactions on Pattern Analysis and Machine Intelligence 11, no. 7, 674-693 (1989).

[12] S. Mallat: A wavelet tour of signal processing. Academic Press (1997).

[13] A. Pizurica, V. Zlokolica, and W. Philips: Noise reduction in video sequences using wavelet-domain and temporal filtering, in SPIE Conference Wavelet Applications in Industrial Processing, Providence, Rhode Island, USA (2003).

[14] R. Ranta, C. Heinrich, V. Louis-Dorr, D. Wolf: Interpretation and improvement of an iterative wavelet-based denoising method. IEEE Signal Processing Letters 10, no. 8, 239-241 (2003).

[15] R. Ranta, C. Heinrich, V. Louis-Dorr, D. Wolf: Iterative wavelet-based denoising methods and robust outlier detection. IEEE Signal Processing Letters 12, no. 8, 557-560 (2005).

[16] R. Ranta, V. Louis-Dorr, C. Heinrich, D. Wolf, F. Guillemin: Digestive activity evaluation by multi-channel abdominal sounds analysis. IEEE Transactions on Biomedical Engineering 57, no. 6, 1507-1519 (2010).

[17] A. van der Vaart, J. Wellner: Weak convergence and empirical processes, Springer (1996).

[18] T. Cai, B. Silverman: Incorporating information on neighbouring coefficients into wavelet estimation, Sankhyā: The Indian Journal of Statistics. Special issue on Wavelets 63, no. 2, 127-148 (2001).

[19] T. Cai, H. Zhou: A Data-Driven Block Thresholding Approach to Wavelet Estimation, The Annals of Statistics, to appear, http://www-stat.wharton.upenn.edu/ tcai/Papers.html

[20] L. Rankine, N. Stevenson, M. Mesbah, B. Boashash: A Nonstationary Model of Newborn EEG, IEEE Transactions on Biomedical Engineering 54, no. 1, 19-28 (2007), http://www.som.uq.edu.au/research/sprcg/newborn.asp

[21] P. Moulin, J. Liu: Analysis of multiresolution image denoising schemes using generalized Gaussian and complexity priors, IEEE Transactions on Information Theory 45, no. 3, 909-919 (1999). 
[22] M. Do, M. Vetterli: Wavelet-Based Texture Retrieval Using Generalized Gaussian Density and Kullback-Leibler Distance, IEEE Transactions on Image Processing 11, no. 2, 146-158 (2002).

[23] R. Buccigrossi, E. Simoncelli: Image compression via joint statistical characterization in the wavelet domain, IEEE Transactions on Image Processing 8, no. 12, 1688-1701 (1999).

[24] E. Simoncelli, R. Buccigrossi: Embedded Wavelet Image Compression Based on a Joint Probability Model, 4th IEEE International Conference on Image Processing - ICIP, Santa Barbara, USA (1997).

[25] A. Pižurica, W. Philips: Estimating the probability of the presence of a signal of interest in multiresolution single- and multiband image denoising, IEEE Transactions on Image Processing 15, no. $3,654-665$ (2006).

Céline Lacaux, Aurélie Muller-Gueudin, Samy Tindel: Institut Élie Cartan, B.P. 239, 54506 Vandœuvrelès-Nancy, France. Email: [lacaux, muller, tindel] @iecn.u-nancy.fr

Radu Ranta: Centre de Recherche en Automatique de Nancy, 2 Avenue de la Fort de Haye, 54500 Vandœuvre-lès-Nancy, France. Email: Radu.Ranta@ensem.inpl-nancy.fr 